Preprints of the

Max Planck Institute for

Research on Collective Goods

Bonn 2010/22

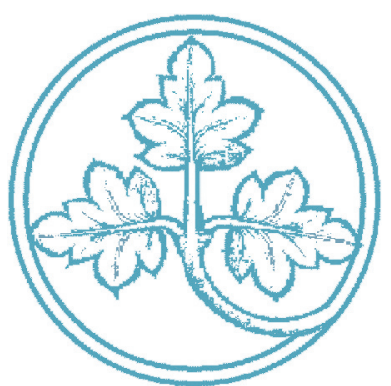

Electoral Competition with Uncertainty Averse Parties

Sophie Bade

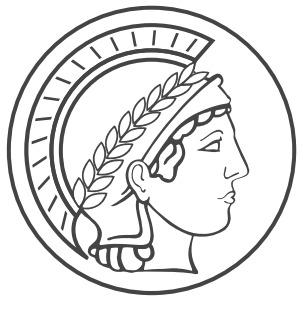




\section{Electoral Competition with Uncertainty Averse Parties}

Sophie Bade

May 2010 


\title{
Electoral Competition with Uncertainty Averse Parties *
}

\author{
Sophie Bade ${ }^{\dagger}$ \\ Max Planck Institute for Collective Goods
}

May 2010

\begin{abstract}
The nonexistence of equilibria in models of electoral competition involving multiple issues is one of the more puzzling results in political economics. In this paper, we relax the standard assumption that parties act as expected utility maximizers. We show that equilibria often exist when parties with limited knowledge about the electorate are modeled as uncertainty-averse. What is more, these equilibria can be characterized as a straightforward generalization of the classical median voter result.
\end{abstract}

Keywords: Uncertainty Aversion, Multiple Priors, Median Voter, Electoral Competition over many Issues. JEL Classification Numbers: D72, D81.

\section{Introduction}

The present paper introduces two interlaced modeling innovations into an otherwise standard model of Downsian electoral competition with a multidimensional policy space. It is, first of all, assumed that electoral outcomes for different constellations of party platforms are subjectively uncertain in the sense that there is no function that maps every constellation of party platforms to objectively known probability distributions over the vote shares for the two parties. Secondly, it is assumed that parties have uncertainty-averse preferences. In justification of the first assumption, observe that real parties do indeed face choices with uncertain consequences. Electoral outcomes are generally hard to predict as elections are

*I would like to thank Jean-Pierre Benoit, Christoph Engel, Alrejandro Jofre, Philip Leifeld, Debraj Ray, Ronny Razin, Ennio Stacchetti, Raquel Fernandez, Alessandro Lizzeri, Philipp Weinschenk and the seminar participants at Columbia University, NYU, the CERMSEM Paris and the Chilean Central Bank. I gratefully acknowledge the hospitality of the Centro de Modelacion Matematica of la Universidad de Chile. My biggest thanks goes out to Efe Ok, my advisor, who tirelessly discussed all the new developments of this work with me.

†bade@coll.mpg.de 
usually held in ever-changing environments involving new issues, turnover of party elites, and an evolving composition of the electorate. Taking the 2008 US presidential election as an example, observe that neither the experience with past elections nor any amount of polling would have been sufficient to determine objective winning probabilities for Barack Obama and John McCain for all possible combinations of platforms, given that they where dealing with some new issues (how to respond to the Lehmann brothers bankruptcy?), a new party elite (Barack Obama was the first African American to be nominated for President by a major political party) and a large share of first-time voters.

The second, related, assumption is that parties are uncertainty-averse, in the sense that all else being equal, parties prefer situations in which they know more about the probability distributions over the vote shares to situations in which there is less clarity about these distributions. Such a bias against uncertainty is evidenced in the famous Ellsberg paradox in which subjects can choose to bet on red or black in either an urn with 5 red and 5 black balls or an urn with a total of 10 red and black balls in unknown proportion. Subjects are generally indifferent between betting on either color for a fixed urn. They do, however, prefer betting on the urn with the known proportion to betting on the other urn. This behavior violates expected utility theory since expected utility-maximizing subjects would have to calculate their preferences according to a fixed probability for red being drawn from the second urn, which has to be either above, below or equal to $1 / 2$. Since such a preference for options involving less uncertainty manifests itself in many experiments (see Camerer and Weber (1992) for a review), we might also expect that the decision makers in parties have uncertainty-averse preferences.

The median voter theorem states that in a game of electoral competition with a unidimensional policy space played by two office motivated parties and a set of voters with single-peaked preferences, both parties will announce the median ideal point in equilibrium (Black 1948, Downs 1957 and Hotelling 1929). The assumption that the policy space is unidimensional is crucial. If one allows for a multidimensional policy space, in a classical Downsian model pure strategy equilibria only exist under very restrictive conditions on the distribution of voter preferences. (Plott (1967), Davis, Hinich and de Groot (1972), Grandmont (1978), McKelvey (1979) all show that a Condorcet winner only exists for very special distributions of voter preferences.) This is problematic since actual electoral campaigns usually concern various different issues and the assumption that all these issues can be aligned perfectly into a unidimensional policy space (say, left to right) is quite strong. Ordeshook (1986) suggests in his discussion of multidimensional games of electoral competition (in his book "Game Theory and Political Theory") that a more realistic modeling approach to the uncertainties of politics might mitigate the problems of equilibrium non-existence. He says that, "We should keep in mind, of course, that our examples do not match reality. Candi- 
dates are uncertain about voters' exact preferences, and voters are only imperfectly informed about candidates' strategies..."

The main results of the present paper validate this claim by Ordeshook: the existence of uncertainty about voters' preferences, together with the assumption that parties are uncertainty-averse, significantly alleviates the problem of equilibrium non-existence in Downsian models with multidimensional policy spaces. It has to be said, though, that the sufficient conditions for equilibrium existence only carry an intuitive interpretation for policy spaces with at most three dimensions. Still, in terms of empirical applicability, this is a major step forward, given that a diverse set of empirical studies finds that actual policy spaces are often of low dimensionality. ${ }^{1}$ In addition to the results on equilibrium existence, the political equilibria in the present model are characterized through a simple extension of the equilibrium prediction of the classic Downsian model to the environment with multiple issues. Thanks to a separability assumption of voter preferences, parties behave as if they were playing separate games of standard Downsian electoral competition in every dimension of the policy space. In equilibrium they do announce the ideal point of some relevant median voters with respect to every dimension.

The driving intuition behind the result of equilibrium existence is that the game of electoral competition played by the parties resembles the Ellsberg paradox in the sense that parties can set their platforms such as to choose between an objective lottery or a subjectively uncertain distribution of vote shares. For a fixed platform of the opponent, a party can eliminate the relevance of all uncertainty on voter preferences over policies by simply announcing the platform of the opponent. Formally, the party generates a situation similar to the urn with the known proportion of balls. Alternatively, the party can announce a platform that differs from the platform of the opponent. In this case, the parties' uncertainty on voter preferences over policies matters for the determination of vote shares for the two parties. Formally, this situation resembles the Ellsberg urn. Consequently the assumption of uncertainty aversion introduces a bias towards adopting the platform of the opponent. If this bias is strong enough to outweigh the desire to deviate from the platform of the other party, equilibrium existence is obtained.

\footnotetext{
${ }^{1}$ The long-term analysis by Poole and Rosenthals long-term analysis of roll call votes finds that a policy space of low dimensionality accurately describes the voting patterns in the US congress (Poole and Rosenthal 1997 and 2007). Hix, Noury, and Roland (2006) apply the same technique to voting in the European Parliament and obtain the same finding: very few dimensions suffice to explain voting patterns of European parliamentarians. Party manifestos provide a different source of information on the issue space. Using data from the Comparative Manifesto Project, Hix (1999) finds two salient policy dimensions for European politics from 1976 until 1994. As an alternative approach to the one of the Comparative Manifesto Project, Laver and Garry (2000) and Laver et al. (2003) use a fully automated method to extract meaning from political texts, to locate the positions of parties. They content themselves with two dimensions each to describe the policy spaces of England, Ireland, and Germany.
} 
There is a range of different proposals on how to represent the preferences of uncertaintyaverse agents, notably Bewley (2002), Gilboa and Schmeidler (1989), Schmeidler (1989), Ghirardato et al. (2003) and Maccheroni et al. (2006). Since the main intuition for the existence result presented in this article is rooted in the Ellsberg paradox, and since all of these approaches are able to explain the Ellsberg paradox, the main arguments of this paper could be based on any of these approaches. Gilboa and Schmeidler's (1989) approach was chosen, since their model allows for tractable results and since it has already found some application outside decision theory. ${ }^{2}$ In political economy, Ghirardato and Katz (2002) use uncertainty aversion to explain the empirical puzzle that voters abstain from elections, even when voting is costless, i.e., when the voter is already in the booth because of a different election. Ashworth (2007) uses a similar framework to explain parties' decisions to target some groups of voters to affect turnout and to target other groups to switch their allegiance. Berliant and Konishi's (2005) explanation of the fact that parties avoid discussing some issues in electoral campaigns is most related to the present paper, as the driving force behind their argument is the assumption that parties are uncertainty-averse.

The assumption that parties are uncertain about electoral outcomes is not new to the literature on electoral competition; models of probabilistic voting do share this assumption with the model of the current paper. ${ }^{3}$ The present model and models of probabilistic voting also share some results: according to both approaches Downsian games of electoral competition with multidimensional policy spaces have equilibria, if the parties' uncertainty about voter behavior satisfies certain assumptions. The two approaches also share the result that both parties announce the same platform in equilibrium. Their assumptions differ insofar as parties are assumed to hold a prior on the behavior of the electorate in models of probabilistic voting, whereas parties in the present model are assumed to be uncertainty-averse. Another major difference lies in the fact that the present model derives the parties' uncertainty about their vote shares from an uncertainty about a primitive of the model, namely the voters' preferences over the policy space as expressed by their ideal points and marginal rates of substitution between the various issues. ${ }^{4}$ Conversely, models of probabilistic voting assume an exogenous source of uncertainly about voter behavior. The models also differ with respect to the location of the equilibrium platform. While the equilibrium platforms in models of probabilistic voting models are calculated as weighted means of the voter ideal points, parties in the present paper announce a median ideal point policy with respect to every dimension of the policy space in equilibrium. In Section 3, the two models will be compared in some more detail.

\footnotetext{
${ }^{2}$ For a survey of applications of the decision theory of uncertainty aversion to various contexts of economics see Mukerji and Tallon (2004).

${ }^{3}$ For reviews of the literature on probabilistic voting, see Coughlin (1992) or Banks and Duggan (2006).

${ }^{4}$ In the literature on probabilistic voting, these models are sometimes called "random utility models".
} 
Other proposals to remedy the problem of equilibrium non-existence in platform positioning games with multidimensional issue spaces include Besley and Coate (1997), Roemer (1999), and Duggan and Jackson (2006). In Besley and Coate's (1997) citizen candidate model, we are faced with the problem that equilibrium sets are potentially very large. The key to equilibrium existence in Roemer (1999) is the assumption that parties have incomplete preferences. In his model, any party consists of three factions called the opportunists, militants and reformists. Parties only deviate from their platform if all three factions unanimously agree that such a deviation implies an improvement for the party. In the context of the present model, an equilibrium would be trivially obtained if each party announced the ideal point of its militant faction, as this faction would veto any deviation from this platform. Roemer avoids such trivial conclusions by imposing some additional assumptions on party preferences that are very reasonable in the context of progressive taxation, the subject of his study. In the very general context of the present study, it would be difficult to identify such additional assumptions. It should be said, however, that our assumption on party preferences could be derived from a similar setup as Roemer's (1999) if one were to attribute the different priors in the utility function of a party to different factions within the party. ${ }^{5}$ Finally, Duggan and Jackson (2006) show that Simon and Zame's (1990) result on endogenous sharing rules can be applied to the case of platform positioning in multidimensional issue spaces. Duggan and Jackson show that mixed strategy equilibria exist in the Downsian model with multiple issues if voters are not assumed to randomize exogenously between two candidates when they are indifferent.

\section{Electoral Competition}

We model electoral competition as a two-stage game played by two political parties, denoted by 1 and 2 , and a set of voters. ${ }^{6}$ First, the two parties simultaneously choose their platforms within some (non-empty) convex policy space $X \subset \mathbb{R}^{d}, d \geq 1$. The separate dimensions of the policy space are called issues. Then the voters, whose preferences are defined over $X$, cast their votes. We assume throughout that parties credibly commit to their proposed policies and that voters only care about policies. In particular, no voter has any ideological

\footnotetext{
${ }^{5}$ Levy (2004) also assumes that parties will only change platforms if the various factions in a party can agree on such a move. Keller, Sarin, and Sounderpandian (2002) provide some empirical evidence that groups act more uncertainty-aversely than individuals, which might be derived from a decision procedure that groups only change their strategies if such a change is judged advantageous from the vantage point of different factions who hold different beliefs about the stochastic environment.

${ }^{6}$ At this moment, I do not specify anything about the size of the electorate, as it is, on the one hand, easier to state introductory examples on the theory with finite electorates and, on the other hand, easier to state and prove theorems for the case of a continuum of voters.
} 
attachment or bias towards either party.

\section{$2.1 \quad$ Voters}

Throughout this paper, we assume that each voter's preferences can be represented by a utility function $u^{g}(\cdot, a): X \rightarrow \mathbb{R}$ with

$$
u^{g}(x, a):=-\sum_{i=1}^{d} g_{i}\left(\left|x_{i}-a_{i}\right|\right) \text { for all } x \in X
$$

where $a \in X, g_{i}: \mathbb{R}_{+} \rightarrow \mathbb{R}_{+}$is a strictly increasing continuous function for all $1 \leq i \leq d$, and $g:=\left(g_{1}, \ldots, g_{d}\right)$. We normalize $g_{i}(0)=0$ for all $i$. Since $u^{g}(x, a)$ is maximized at $x=a$, we call the vector $a \in X$ the voter's ideal point. Observe that the functions $u^{g}\left(\left(\cdot, x_{-i}\right), a\right)$ are single-peaked for any $x_{-i} \in X_{-i}$. In fact, any preference relation that can be represented by a function $u^{g}(\cdot, a)$ that is single-peaked according to the most common notions of singlepeaked ness for multidimensional issue spaces which require that utility monotonically falls on every arrow that points away from the ideal point of the voter (cf. Barbera, Gul, and Stachetti (1993) and Roemer (2001)). Since the vector $g$ determines the form of a voter's indifference curves, we refer to it as the shape of the voter's indifference curves and we call the function $g_{i}$ the attitude of a voter towards issue $i$. Geometrically, the indifference curves of two voters with the same shape are translations of each other.

Observe that the model in which a voter's utility of a policy is a negative transformation of the Euclidean distance between his ideal point and that policy is a special case, of the present model. To obtain this special case we need to set $g_{i}(t):=t^{2}$ for all $t \in \mathbb{R}_{+}$and $1 \leq i \leq d$; we denote this shape by $g^{\circ}$. The shape $g$ for which $g_{i}(t):=\alpha_{i} t$ for some $\alpha_{i}>0$ for all $1 \leq i \leq d$ will play a major role in this paper. We denote such a shape by the vector $\alpha:=\left(\alpha_{1}, \ldots, \alpha_{d}\right)$. The indifference curves that correspond to utility function $u_{\alpha}(\cdot, a)$ are diamond-shaped. Notably, for such a voter the marginal rate of substitution between all issues is constant.

\section{Insert Figure 1}

Formally speaking, a voter in the present setup is characterized by a pair

$$
(a, g) \in X \times H
$$

where $H$ is some finite set of shapes $H:=\left\{g^{1}, \ldots, g^{m}\right\}$ with $g^{j}=\left(g_{1}^{j}, \ldots, g_{d}^{j}\right)$ and each $g_{i}^{j}$ being some strictly increasing continuous map on $\mathbb{R}_{+}$. Consequently, the entire electorate 
can be described by a distribution of voters $(a, g)$, which is modeled as a Borel probability measure on $X \times H$. Thus, if we denote the set of all Borel probability measures on a metric space $Y$ by $\mathbb{P}(Y)$, then the space of all possible electorates in this model is $\mathbb{P}(X \times H)$. For any $\psi \in \mathbb{P}(X \times H)$, we define the distribution of voter ideal points $\psi_{a} \in \mathbb{P}(X)$ and the distribution of shapes $\psi_{g} \in \mathbb{P}(H)$ as the marginal distributions of $a$ and $g$, respectively. That is,

$$
\psi_{a}(A):=\psi(A \times H) \text { and } \psi_{g}(K):=\psi(X \times K),
$$

for any Borel sets $A$ in $X$ and $K$ in $H$.

The expression $\pi_{\psi}(x, y)$ denotes the share of voters that will vote for platform $x$ given that the other platform is $y$ and given that the electorate is described by $\psi$. We assume that that this share $\pi_{\psi}(x, y)$ can be calculated as the total mass of all voters who strictly prefer $x$ to $y$ plus half the mass of all indifferent voters. More formally, we can express the vote share-function $\pi_{\psi}: X \times X \rightarrow[0,1]$ by

$$
\pi_{\psi}(x, y):=\psi(T(x, y))+\frac{1}{2} \psi(I(x, y)) .
$$

with

$$
T(x, y):=\left\{(a, g) \in X \times H: u^{g}(x, a)>u^{g}(y, a)\right\}
$$

and

$$
I(x, y):=\left\{(a, g) \in X \times H: u^{g}(x, a)=u^{g}(y, a)\right\}
$$

for any $(x, y) \in X \times X$, the sets of voters that strictly prefer $x$ to $y$ and the set of indifferent voters, respectively. The vote share function $\pi_{\psi}$ is well defined, since both $T$ and $I$ are measurable. The following example provides a concrete illustration of the model presented so far.

Example 1: Consider a world in which there are 5 voters and 2 issues. The policy space $X$ equals $[0,1]^{2}$. There are two voters with ideal point $\left(\frac{1}{2}, \frac{1}{2}\right)$ and shape $\alpha^{*}:=(1,1)$. There is one voter with ideal point $(0,0)$ and circular indifference curves, two other voters with the same ideal point $\left(\frac{2}{3}, \frac{1}{3}\right)$ but different shapes, one has shape $\alpha^{*}$ and the other has circular indifference curves. This electorate is formally described by the distribution $\psi \in \mathbb{P}\left([0,1]^{2} \times\left\{g^{\circ}, \alpha^{*}\right\}\right)$ with $\psi\left(\left\{\left(\left(\frac{1}{2}, \frac{1}{2}\right), \alpha^{*}\right)\right\}\right)=\frac{2}{5}, \psi\left(\left\{\left((0,0), g^{\circ}\right)\right\}\right)=\psi\left(\left\{\left(\left(\frac{2}{3}, \frac{1}{3}\right), \alpha^{*}\right)\right\}\right)=$ $\psi\left(\left\{\left(\left(\frac{2}{3}, \frac{1}{3}\right), g^{\circ}\right)\right\}\right)=\frac{1}{5}$. Now suppose the two parties propose the platforms $x=\left(0, \frac{1}{3}\right)$ and $y=\left(\frac{5}{6}, 0\right)$, respectively. In this case, we have that $\pi_{\psi}(x, y)=\psi\left(\left(\frac{1}{2}, \frac{1}{2}\right), \alpha^{*}\right)+\psi\left((0,0), g^{\circ}\right)=\frac{3}{5}$.

\section{Insert Figure 2}

The dotted lines in Figure 2 represent the indifference curves of the voters $\left(\left(\frac{2}{3}, \frac{1}{3}\right), \alpha^{*}\right)$ and $\left(\left(\frac{2}{3}, \frac{1}{3}\right), g^{\circ}\right)$ through their preferred platform $\left(\frac{5}{6}, 0\right)$. The dashed arc represents the indifference curve of a voter $\left((0,0), g^{\circ}\right)$ through his preferred platform $\left(0, \frac{1}{3}\right)$. The remaining (bold) lines all belong to the indifference curve of voter $\left(\left(\frac{1}{2}, \frac{1}{2}\right), \alpha^{*}\right)$ through his preferred platform: $\left(0, \frac{1}{3}\right)$. 


\subsection{Parties}

The goal of each party is to maximize its vote share. ${ }^{7}$ A party's strategy variable is its platform, and hence $X$ is its strategy space. If the electorate were known, the objective of a party would simply be to maximize $\pi_{\psi}(x, y)$. Parties in our model, however, do not know the electorate; they are instead uncertain about voters' preferences. The innovation of this paper is to study such an environment under the hypothesis that parties are uncertainty-averse.

In particular, following Gilboa and Schmeidler (1989) we assume that party preferences can be represented with Maxmin expected utilities. The preferences of party i can be represented by a map $\Pi_{\mathcal{P}_{i}}: X \times X \rightarrow \mathbb{R}$ with

$$
\Pi_{\mathcal{P}_{i}}(x, y):=\min _{p \in \mathcal{P}_{i}} \int_{\mathbb{P}_{i}(X \times H)} \pi_{\psi}(x, y) p(d \psi),
$$

where $\mathcal{P}_{i} \subseteq \mathbb{P}(\mathbb{P}(X \times H))$ is a non-empty, convex and compact set of priors on the electorate $\psi$ and where $x$ denotes the platform of Party 1 and $y$ the other party's platform (exchanging the names, we obtain Party 2's preferences). Note that in this model the set of all electorates $\mathbb{P}^{*}(X \times H)$ represents the set of all possible states. Keeping the platform of Party 2 fixed at some $y \in X$, Party 1 can choose any platform $x$ in $X$. Each of these platforms $x$ corresponds to an act $f_{x}$ assigning to every state $\psi$ a consequence $\pi_{\psi}(x, y)$. Formally the assumption that parties are vote share maximizers translates to the assumption that Party 1 derives utility $\pi_{\psi}(x, y)$ from the consequence $\pi_{\psi}(x, y)$, so the function $\psi \mapsto \pi_{\psi}(x, y)$ represents the utility of Party 1 in each state. A generic element of the set $\mathbb{P}\left(\mathbb{P}^{*}(X \times H)\right)$ is viewed as a belief (or prior) on the electorate; it is a probability measure on the set of all electorates (or states) $\mathbb{P}^{*}(X \times H)$. The case of expected vote share maximizing parties is embedded for the set of party beliefs being a singleton.

Schmeidler's (1989) definition of uncertainty aversion requires that an agent who is indifferent between two acts (weakly) prefers a mixture of these two acts to either one of them. In terms of the game of electoral competition the concept of uncertainty aversion can be expressed as follows. Suppose in some election Party 2 runs on platform $y$ and Party 1 is indifferent between opposing $y$ with platform $x$ or platform $x^{\prime}$. Now let that party go through a thought experiment in which the party imagines that same identical election twice. In each of these identical elections, Party 2 runs on platform $y$. Then, by the uncertainty aversion axiom of Schmeidler, Party 1 must like to offer $x$ in one of the elections and $x^{\prime}$ in the other at least as much as offering $x$ in both elections. In short, we assume that if a party had a

\footnotetext{
${ }^{7}$ This assumption on the preferences of parties is standard, but not without criticism. The other most common assumption on party preferences is that they maximize their probability of winning. The true goal of a party is likely a convex combination of these two. We chose the assumption of vote share maximization since it allows for some neat simplifications of the parties' objective functions.
} 
chance to hedge its bets, it would not reject this chance.

\subsection{The Game}

The game with the two parties as players, $X$ as each party's strategy space and $\Pi_{\mathcal{P}_{i}}: X \times X \rightarrow$ $\mathbb{R}$ as the payoff function of parties $i=1,2$ is called a game of electoral competition. A (pure) strategy profile $(x, y)$ is called a political equilibrium if it constitutes a Nash equilibrium in the game of electoral competition. ${ }^{8}$

We provide an example of a game of electoral competition that does not have an equilibrium when one assumes that parties are expected utility maximizers. We show that when one assumes that parties are sufficiently uncertain about the electorate and sufficiently uncertainty-averse, this same game does have a political equilibrium.

Example 2: Consider the game of electoral competition with 3 voters, and a 2 dimensional issue space $X=[-1,1]^{2}$. Assume that there are two states of the world, $\psi_{1}$ and $\psi_{2}$. Assume that, independently of the state, all voters have Euclidean preferences and voters 1 and 2 have their ideal points at $(-1,-1)$ and $(1,1)$, respectively. Assume, furthermore, that voter 3's ideal point depends on the state of the world. In state 1 , it is $(-1,1)$; in the alternative state, it is $(1,-1)$. Now let both parties be expected utility maximizers and assume that they believe that state 1 obtains with a probability of $\frac{5}{8}$. To see that this game has no equilibrium, suppose the platform profile $(x, y)$ does constitute a political equilibrium. Observe that in equilibrium each party has to get an expected vote share of $\frac{1}{2}$. Next, observe that for any platform of Party 2, and therefore also for platform $y$, there exists a platform $x^{\prime}$, for Party 1 such that Party 1 obtains at least two votes in state 1 . Since the preferences of voters 1 and 2 are not state-dependent, this means also that Party 1 obtains at least one vote in state 2 given the platform profile $\left(x^{\prime}, y\right)$. Combining these two observations with the modeling assumption that parties agree that state 1 happens with probability $\frac{5}{8}$, we can conclude that Party 1 always has a deviation according to which its expected vote share is $\frac{1}{3}\left(\frac{5}{8} 2+\frac{3}{8} 1\right)>\frac{1}{2}$. No political equilibrium exists.

Now consider the case that parties are uncertainty-averse and that their preferences can be represented following Gilboa and Schmeidler. Assume that both parties base the calculation of their maxmin expected vote shares on a set of priors $\left[\frac{4}{9}, \frac{2}{3}\right]$ which is to be interpreted as the set of probabilities that state 1 obtains $\left(p\left(\psi_{1} \in\left[\frac{4}{9}, \frac{2}{3}\right]\right)\right)$. Consider the strategy profile

\footnotetext{
${ }^{8}$ Since the interpretation of mixed strategies poses a challenge in the context of games of electoral competition, we do not examine mixed strategy equilibria in the present paper. In addition it has to be said that the extension of the present model to one in which parties are allowed to mix is non-trivial: one would have to make some well-founded choices on the interaction between the endogenous uncertainty (mixtures of parties) and the exogenous uncertainty about the voters' preferences.
} 
according to which both parties offer the platform $(0,0)$. Observe that, for any deviation from this platform profile, the deviator party obtains at most two votes in one of the two states and one vote for the other state. A party that ponders a deviation that yields more votes in $\psi_{1}$ uses $p\left(\psi_{1}\right)=\frac{4}{9}$ to calculate its maxmin expected vote share as smaller or equal than $\frac{1}{3}\left(\frac{4}{9} 2+\frac{5}{9}\right)<\frac{1}{2}$. Conversely, if the deviation under consideration yields more votes in $\psi_{2}$ the maxmin expected vote share is smaller or equal to $\frac{1}{3}\left(\frac{1}{3} 2+\frac{2}{3}\right)<\frac{1}{2}$. In sum, there are no profitable deviations from $((0,0)(0,0))$; the profile is a political equilibrium.

It is important to note that it is conceptually impossible to consider the "same model" with and without uncertainty aversion. In the example, parties were once modeled as expected utility maximizers with a prior of $\frac{5}{8}$ and once as maxmin expected utility maximizers with a set of priors $\left[\frac{4}{9}, \frac{2}{3}\right]$. However, nothing forces us to assume this particular prior and this particular set of priors. If the expected utility maximizing parties had a single prior $p$ with $p\left(\psi_{1}\right)=\frac{1}{2}$, the profile $((0,0),(0,0))$ would also be an equilibrium for expected utility maximizing parties. Conversely if the uncertainty-averse parties had a set of priors $\left[\frac{1}{8}, \frac{1}{4}\right]$, no political equilibrium would exist even for the uncertainty-averse parties. It is important to note, though, that the case in which an equilibrium exists for expected utility-maximizing parties is a knife-edge case: an equilibrium exists if and only if $p\left(\psi_{1}\right)=\frac{1}{2}$. Conversely, the set of sets of beliefs for which an equilibrium exists is a large one, namely a political equilibrium exists for all intervals $[a, b]$ with $a \leq \frac{1}{2} \leq b$.

Consequently, the claim made by the present paper is not that uncertainty aversion suffices to obtain equilibrium existence in games of electoral competition. Rather, the main claim is that while the conditions under which equilibria exist in multidimensional games of electoral competition with expected vote share maximizing parties are knife-edge conditions, the conditions for equilibrium existence for the case of uncertainty-averse parties are rather generic - at least as long as one considers only two- to three-dimensional policy spaces.

\section{Large Electorates}

While it is easier to state concrete examples in terms of finite electorates, as was done above, the derivation of general results turns out to be easier when assuming non-atomic distributions of voter ideal points, since in this case medians of distributions of most preferred policies with respect to single issues are unique. We therefore impose from now on that parties believe that the voter ideal point distribution is non-atomic and has full support. Formally, we assume that for any $\psi \in \operatorname{supp}(p)$ for some $p \in \mathcal{P}$, the distribution of voter ideal points $\psi_{a}$ is non-atomic and $\operatorname{supp}\left(\psi_{a}\right)=X$. We write the set of all Borel measures fulfilling these additional assumptions as $\mathbb{P}^{*}(X \times H)$. In the context of large electorates, this seems to be a reasonable simplification. 
It turns out that it is equivalent to think of the decision problem of parties in terms of expected vote shares or in terms of vote shares according to the expected electorate. To this end, the set of expected voter distributions for a set of beliefs on the electorate $\mathcal{P} \in \mathbb{P}\left(\mathbb{P}^{*}(X \times H)\right)$ is defined as $\Psi:=\left\{\int_{\mathbb{P}^{*}(X \times H)} \psi p(d \psi): p \in \mathcal{P}\right\}$.

Proposition 1: For any non-empty, convex and compact subset $\mathcal{P}$ of $\mathbb{P}\left(\mathbb{P}^{*}(X \times H)\right)$, the set $\Psi:=\left\{\int_{\mathbb{P}^{*}(X \times H)} \psi p(d \psi): p \in \mathcal{P}\right\}$ is a convex subset of $\mathbb{P}^{*}(X \times H)$, and

$$
\Pi_{\mathcal{P}}(x, y)=\min _{\psi \in \Psi} \pi_{\psi}(x, y) \quad \text { for all }(x, y) \in X^{2}
$$

Proof: See Appendix.

Proposition 1 shows that it is without loss of generality to represent the preferences of Party 1 over platform profiles by a map of the form:

$$
(x, y) \mapsto \min _{\psi \in \Psi} \pi_{\psi}(x, y)
$$

where $\Psi$ is some non-empty convex subset of $\mathbb{P}^{*}(X \times H)$. Moreover, it will simplify things greatly to view $\Psi$ as the primitive of the model and to introduce our further assumptions on party beliefs, as assumptions on this set.

Remark: Expected vote share maximizing parties arise as a special case of multiple-prior formulation of party preferences. If the set of priors $\mathcal{P}$ is a singleton, say $\left\{p^{*}\right\}$, the objective of any party is to maximize its expected vote share. In this case, the utility of Party 1 reduces to

$$
\Pi_{\mathcal{P}}(x, y)=\int_{\mathbb{P} *(X \times H)} \pi_{\psi}(x, y) p^{*}(d \psi) .
$$

Following Proposition 1, this is exactly the vote share of Party 1 when the platform profile is $(x, y)$ and the expected electorate $\psi^{*}:=\int_{\mathbb{P}^{*}(X \times H)} \psi p(d \psi)$. That is,

$$
\Pi_{\left\{p^{*}\right\}}(x, y)=\pi_{\psi^{*}}(x, y)
$$

where $\psi^{*}(S):=\int_{\mathbb{P}^{*}(X \times H)} \psi(S) p(d \psi)$ for all Borel subsets $S$ of $X \times H$. Therefore, models with parties that are certain about the electorate and models with expected vote share maximizing parties are formally equivalent. Consequently, there is no hope to solve the well-known nonexistence problems of the standard electoral competition models with multidimensional issue spaces by just introducing risk (or Savagean uncertainty) into these models. In other words, this is a formal statement and proof of Banks and Duggan's (2006) assertion of a "folk theorem" on probabilistic voting which is that the "Random Utility Model" (deriving party 
uncertainty on vote shares from their uncertainty about voter types) is formally equivalent to the model of electoral competition with parties that know the voter distribution. ${ }^{9}$ To obtain equilibrium existence probabilistic voting models have to assume that the uncertainty about vote shares derives from an exogenous source, not from uncertainty about aspects of voter preferences.

To save on notation, we let $\Psi_{1}=\Psi_{2}=\Psi$. This assumption is not needed for any of the results in the paper: all results hold if the conditions on $\Psi$ are instead applied to each of the set of beliefs $\Psi_{1}, \Psi_{2}$. The set $\Psi$ not only reflects the parties' beliefs about the electorate but it also reflects their uncertainty aversion. Therefore it is not appropriate to invoke the common prior assumption as a justification of the assumption that $\Psi_{1}=\Psi_{2}=\Psi$. Two actors that have access to the same amount of information might base their decisions on different sets of priors, simply because actors' attitudes towards uncertainty might differ. There are some decision-theoretic models that distinguish between the uncertainty of the decision maker and his attitude towards that uncertainty; see, for instance, Ghiradato (2004) and Klibanoff, Marinacci, and Mukerji (2005). Additional comments on the common prior assumption can be found in Remark (d) in Section 4.1.

We need further assumptions on the nature of the set $\Psi$ of parties' beliefs to establish our main results. To this end, we define the set $\Psi(\Lambda, G)$ as the set of beliefs on the electorate that has been generated by the set $\Lambda$ of voter ideal point distributions and by the set $G$ of shapes as follows:

$$
\Psi(\Lambda, G):=\left\{\psi \in \mathbb{P}^{*}(X \times H): \psi_{a} \in \Lambda \text { and } \operatorname{supp}\left(\psi_{g}\right) \subseteq G\right\}
$$

This definition says that for any electorate in $\Psi(\Lambda, G)$, the voter ideal point distribution belongs to $\Lambda$ and the shape of the indifference curves of any voter belongs to set $G$. Our assumption states that party beliefs about the electorate can be generated from a set of beliefs on the voter ideal point distribution and some sets of attitudes towards all the different issues.

(A1) There exists a non-empty, convex set $\Lambda \subset \mathbb{P}(X)$ and non-empty, finite sets $G_{i}$ of strictly increasing and continuous maps on $\mathbb{R}_{+}$such that $\Psi=\Psi\left(\Lambda, G_{1} \times \cdots \times G_{d}\right)$.

(A1) excludes the case that certain attitudes about issues say $g_{1}^{\prime}$ only arise in combination with certain other attitudes about other issues, say $g_{2}^{\prime}$ or $g_{2}^{\prime \prime}$. In contrast, if according to some

\footnotetext{
${ }^{9}$ Duggan (2007) notes in a somewhat different context that "the probabilistic voting model [in which parties share a prior about the distribution of voter preferences], once majority preferences have been generated, is formally a special case of the general continuum model" in which parties maximize expected vote shares given a known electorate. I chose to provide my own proof of Proposition 1, since Banks and Duggan (2006) do not formally state and prove the claimed "folk theorem", and since Duggan's (2007) proof concerns a slightly different context.
} 
belief the indifference curves of some voters have the shape $\left(g_{1}, g_{2}\right)$ (over a two-dimensional issue space) while others have the shape $\left(g_{1}^{\prime}, g_{2}^{\prime}\right)$, then there must be an alternative belief in the set $\Psi$ according to which some voters have indifference curves with shape $\left(g_{1}^{\prime}, g_{2}\right)$. (A1) also excludes the case that parties associate certain sets of ideal points with a particular type of indifference-curve shape. (A1) requires that if for some $\psi \in \Psi$ the parties believe that all voters with ideal points in some set $A \subset X$ have the shape $g$, then there must be a $\psi^{\prime} \in \Psi$ such that parties believe that all voters with ideal point not in $A$ have that shape $g$.

The standard case of an electorate where all voters are assumed to have Euclidean preferences fulfils (A1), as can be seen by letting $G_{i}$ consist only of the map $t \mapsto t^{2}$ for all $i$. The belief set of parties that are maximally uncertain about the electorate also satisfies (A1) as we have that $\mathbb{P}^{*}(X \times H)=\Psi\left(\mathbb{P}^{*}(X), H\right)$. More importantly, given (A1), we can control the amount of uncertainty in the model, by varying the sizes of $\Lambda$ and $G$. For instance, take a set of belief sets $\Psi^{\nu}:=\Psi\left(\Lambda^{\nu}, G^{\nu}\right)$ for a sequence of sets $\left(\Lambda^{\nu}, G^{\nu}\right)_{\nu \in \mathbb{N}}$. Let $\Lambda^{\nu} \supset \Lambda^{\nu+1}$ and $G^{\nu} \supset G^{\nu+1}$ for all $\nu \in \mathbb{N}$, which of course implies that $\Psi^{\nu} \supset \Psi^{\nu+1}$ for all $\nu \in \mathbb{N}$. Then the uncertainty of parties decreases as $\nu$ becomes larger. Consequently, the degree of uncertainty can be varied continuously within the model. Electorates in which all voters have the same shape of indifference curves $g=\alpha$ play a major role in the proofs of this paper. Such an electorate is denoted by $\mu \times \alpha$ with the understanding that $\mu$ is the voter ideal point distribution and $\alpha$ the (diamond-)shape of all voter's indifference curves.

Under Assumption (A1), all the information that defines a game of electoral competition is summarized by the quadruple $(d, X, \Lambda, G)$. Both players share the $d$ dimensional policy space $X$ as their action space. The players' payoff functions are derived from the set of beliefs on the distribution of the electorate $\Lambda$ and the set of beliefs on voter types $G=G_{1} \times \cdots \times G_{n}$, as follows:

$$
\min _{\pi \in \Psi(\Lambda, G)} \pi_{\psi}(x, y) \quad \text { and } \min _{\pi \in \Psi(\Lambda, G)}\left(1-\pi_{\psi}(x, y)\right) \quad \text { for all }(x, y) \in X^{2} .
$$

Since the quadruple $(d, X, \Lambda, G)$ uniquely defines a game of electoral competition, it is convenient to call this quadruple itself the game of electoral competition.

We conclude this section with a Lemma about two preliminary observations on games of electoral competition. The first one is that in any political equilibrium, both parties have to get exactly half the vote share according to all beliefs on the electorate. The second is that an increase in the amount of uncertainty and uncertainty aversion in a game of electoral competition, as reflected by the increase of the sets $\Lambda$ and $G$, weakly increases the set of equilibria.

Lemma 1. Let $(d, X, \Lambda, G)$ and $\left(d, X, \Lambda^{\prime}, G^{\prime}\right)$ be two games of electoral competition with $\Lambda \subset \Lambda^{\prime}$ and $G \subset G^{\prime}$ and let $(x, y)$ be political equilibrium of the former game. Then $\pi_{\psi}(x, y)=$ $\frac{1}{2}$ for all $\psi \in \Psi(\Lambda, G)$ and $(x, y)$ is also an equilibrium of the latter game. 
Proof: Let $(x, y)$ be an equilibrium in $(d, X, \Lambda, G)$. Suppose we had that $\pi_{\psi^{\prime}}(x, y)<\frac{1}{2}$ for some $\psi^{\prime} \in \Psi(\Lambda, G)$. Then $\min _{\psi \in \Psi(\Lambda, G)} \pi_{\psi}(x, y)<\frac{1}{2}$ and Party 1 would be better off to offer $y$ as $\pi_{\psi}(y, y)=\frac{1}{2}$ for all $\psi \in \Psi(\Lambda, G)$ and therefore $\min _{\psi \in \Psi(\Lambda, G)} \pi_{\psi}(y, y)=\frac{1}{2}$. If, on the other hand, $\pi_{\psi^{\prime}}(x, y)>\frac{1}{2}$ for some $\psi^{\prime} \in \Psi(\Lambda, G)$, then Party 2 would like to offer $x$ by the same reasoning. To see that $(x, y)$ is also an equilibrium of the latter game, observe that neither player has a profitable deviation from $(x, y)$ as $\min _{\psi \in \Psi(\Lambda, G)} \pi_{\psi}(x, y)=\frac{1}{2} \geq$ $\min _{\psi \in \Psi(\Lambda, G)} \pi_{\psi}(x, y) \geq \min _{\psi \in \Psi\left(\Lambda^{\prime}, G^{\prime}\right)} \pi_{\psi}(x, y)$ where the first inequality follows from $(x, y)$ being an equilibrium in the former game and the second following from $\Lambda^{\prime}$ and $G^{\prime}$ being supersets of $\Lambda$ and $G$.

\section{Uncertainty about Marginal Rates of Substitution}

The voters' marginal rates of substitution between issues are determined by the shapes of their indifference curves $g$. Consequently, the parties' uncertainty about the voters' rates of substitution between the issues can be expressed as uncertainty about the shapes of indifference curves. Information about all voters' patterns of marginal rates of substitution between issues is probably even harder to obtain than information about their ideal points; we therefore start out by analyzing games of electoral competition when parties are only uncertain about rates of substitution and show that any game of electoral competition with two issues has a political equilibrium if parties are uncertain as to whether the voters' marginal rates of substitution between these two issues is more or less than some fixed rate. This includes games with known distributions of voter ideal points. So we can say that for games of electoral competition with only two issues, one kind of uncertainty is sufficient for the existence of political equilibria. Such equilibria exist even if parties are only uncertain about the voters' marginal rates of substitution. The following definition of uncertainty about the marginal rates of substitution comprises the case of $n$ issues.

Definition: A party is (strictly) uncertain about the marginal rates of substitution if there exists an $\alpha \in \mathbb{R}_{++}^{d}$ such that in all $G_{i}$ there are some differentiable functions $h_{i}$ and $k_{i}$ with $h_{i}^{\prime}(t) \geq \alpha_{i} \geq k_{i}^{\prime}(t)$ for all $t \in \mathbb{R}_{+}$(or, in the case of strict uncertainty, $\left.h_{i}^{\prime}(t)>\alpha_{i}>k_{i}^{\prime}(t)\right)$.

To illustrate this condition, fix a platform $x$ and consider a voter with ideal point $a$. A party that is uncertain about the marginal rates of substitution does not know that voter's indifference curve through platform $x$. It does, however, have a fixed rate of substitution $\frac{\alpha_{1}}{\alpha_{2}}$ in mind and always takes the cases that the rate of substitution is larger or smaller than this rate into account. Formally, the assumption that parties are uncertain about the shapes of voter indifference curves $g$ is equivalent to the assumption that voters have thick indifference 
curves and each party assumes that all indifferent voters and all voters with a strict preference for the other party will vote for the other party. In terms of these thick indifference curves, the assumption that parties are (strictly) uncertain about the marginal rates of substitution arises if there exists a shape $\alpha$ such that every one of these thick indifference curves contains the indifference curve of that shape $\alpha$.

This definition is not vacuous. If all voters have Euclidean preferences, the condition is, for example, not fulfilled. Observe, on the other hand, that parties need not be very uncertain for this condition to be fulfilled. Indifference curves do not have to be very thick to contain the indifference curves of shape $\alpha$. Parties might, for example, know that the marginal rate of substitution between issues $i$ and $j$ lies in a small interval around $\frac{\alpha_{i}}{\alpha_{j}}$.

In fact, the extreme case that parties know that all voters' indifference curves have the same shape $\alpha$ is also covered by the definition of uncertainty about the marginal rates of substitution. For such voters, the marginal rates of substitution between all issues are constant over the entire range of platforms. Our definition of uncertainty about marginal rates of substitution still applies, even though, in this case, the set $G$ and therefore also the set $\Psi(\{\mu\}, G)$ are singletons.

The following Theorem states that any two-dimensional game with parties that are uncertain about the marginal rates of substitution of voters has an equilibrium. Even these in which parties know the distribution of voter ideal points $\mu$. As long as there are only two issues involved, the uncertainty about marginal rates of substitution is sufficient for the existence of equilibria. Of course, following Lemma 1, two-dimensional games of electoral competition also have equilibria if parties are in addition uncertain about $\mu$.

Theorem 1. Let $(2, X,\{\mu\}, G)$ be a game where parties are uncertain about the marginal rates of substitution. Then $(2, X,\{\mu\}, G)$ has a political equilibrium.

There is no hope of demonstrating this theorem using Nash's Existence Theorem or any of its relatives, for the best response correspondences in games of the type $(2, X,\{\mu\}, G)$ are generally not convex-valued. We proceed by means of a different strategy: we first show that there is only one candidate for an equilibrium, and then prove that in our game there does not exist any preferred deviation for either party from that platform profile.

\subsection{Characterization of Political Equilibria}

To characterize the set of equilibria of a game of electoral competition $(d, X,\{\mu\}, G)$, we need to introduce the notion of the median vector. For any probability distribution $\mu$, we call the vector of the medians of all marginal distributions $\mu_{i}$, that is, the vector $\mathbf{m}(\mu):=$ $\left(m\left(\mu_{1}\right), \ldots, m\left(\mu_{d}\right)\right)$, the median vector of $\mu$. Since $\mu$ has full support on the convex set 
$X$, the medians of the marginal distributions and thereby the median vector are uniquely defined. Throughout this section, we normalize the median vector of $\mu$ to $\mathbf{0}: \mathbf{m}(\mu)=\mathbf{0}$, and take the convention that $\mathbf{0}$ denotes the vector $(0,0, \ldots, 0)$ (the dimension of this vector will always be clear from the context).

Observe that the median vector can be found as the intersection of $n$ hyperplanes such that each of them cuts the policy space $X$ into two parts containing exactly half of the probability mass of $\mu$ and has one of the $n$ base vectors as its normal vector. These $n$ hyperplanes always intersect and therefore the median vector always exist. Note that the generalized median of $\mu$ is defined as a point such that every hyperplane running through the generalized median cuts the policy space $X$ into two parts containing exactly half of the probability mass of $\mu$. This is a strong requirement; not every distribution has a generalized median. However, if a distribution $\mu$ does have a generalized median, it must coincide with the median vector of the distribution.

Proposition 2. Let $(x, y)$ be an equilibrium of the game $(d, X,\{\mu\}, G)$, then $x=y=\mathbf{0}$.

Proof: Suppose that $(x, y) \neq(\mathbf{0}, \mathbf{0})$, say $y_{1} \neq 0$. Let $x^{\prime}:=\left(0, y_{2}, \ldots, y_{d}\right)$. Facing the choice between $x^{\prime}$ and $y$, all voters decide on the basis of whether $g_{1}\left(\left|0-a_{1}\right|\right)$ is smaller or larger than $g_{1}\left(\left|y_{1}-a_{1}\right|\right)$. Since 0 is median of $\mu_{1}$, at least half of the voters will vote for $x^{\prime}$. And since $\operatorname{supp}(\mu)$ is convex and since $G_{1}$ is a finite set of continuous functions, we have $\pi_{\psi}\left(x^{\prime}, y\right)>\frac{1}{2}$ for all $\psi \in \Psi(\{\mu\}, G)$. Consequently, $\min _{\psi \in \Psi(\{\mu\}, G)} \pi_{\psi}\left(x^{\prime}, y\right)>\frac{1}{2}$. Since $x$ is a best response to $y$, in equilibrium Party 1 receives a utility of $\min _{\psi \in \Psi(\{\mu\}, G)} \pi_{\psi}(x, y)>\frac{1}{2}$. Therefore, Party 2 only receives a utility of $\min _{\psi \in \Psi(\{\mu\}, G)}\left(1-\pi_{\psi}(x, y)\right)<\frac{1}{2}$ in equilibrium. But then Party 2 would be strictly better off by changing its platform to $x$ since $\min _{\psi \in \Psi(\{\mu\}, G)}\left(1-\pi_{\psi}(x, x)\right)=\frac{1}{2}$.

The intuition behind Proposition 2 is that any party can choose to compete with the other in only one dimension. The only protection against such "unidimensional attacks" is to propose the median $m\left(\mu_{i}\right)=0$ associated with every issue $i$. Also, observe that the certainty case is covered by Proposition 2: if $G$ is a singleton, then parties know the distribution of voter ideal points $\mu$ as well as the shape of voters' indifference curves $G=\{g\}$. Given that we now know that $(\mathbf{0}, \mathbf{0})$ is the only strategy profile that could possibly be an equilibrium, we can now approach the problem of equilibrium existence as the question when this particular strategy profile is an equilibrium.

\subsection{The Existence of Political Equilibrium}

A sufficient condition for Theorem 1 to hold is that for any deviation $y$ from the median vector $\mathbf{0}$, there exists an electorate $\psi^{y}$ in the set of electorates $\Psi(\{\mu\}, G)$, such that $\pi_{\psi^{y}}(y, \mathbf{0})<$ 
$\frac{1}{2}$. If there is one such electorate, then $\min _{\psi \in \Psi(\{\mu\}, G)} \pi_{\psi}(y, \mathbf{0})<\frac{1}{2}$ while $\min _{\psi \in \Psi(\{\mu\}, G)} \pi_{\psi}(\mathbf{0}, \mathbf{0})=$ $\frac{1}{2}$. Uncertainty aversion proves a strong force towards both parties announcing the same platform. The entire uncertainty is eliminated when both parties announce the same platform since in this case they both receive half the vote under any assumption on the electorate.

The complete proof that such a $\psi^{y}$ exists can be found in the appendix. Here we give a sketch of the basic argument. First, we show that, given our assumption that parties are uncertain about the marginal rates of substitution, for any platform $y \in X$ there exists some $\psi^{y} \in \Psi(\{\mu\}, G)$ such that $\pi_{\psi^{y}}(y, \mathbf{0}) \leq \pi_{\mu \times \alpha}(y, \mathbf{0})$ where $\alpha$ is chosen such that there exist $h_{i}, k_{i} \in G_{i}$ with $h_{i}^{\prime}(t) \geq \alpha_{i} \geq k_{i}^{\prime}(t)$ for all $t \in \mathbb{R}_{+}$and $i=1$, 2. So while the shape $\alpha$ itself might not be contained in $G$, for any $y$ there exists some electorate $\psi \in \Psi(\{\mu\}, G)$ according to which the vote share of Party 1 is no higher than what it would get if all voters had shape $\alpha$. In fact, this holds independently of the dimension of the issue space of the game, and we conclude that whenever $(\mathbf{0}, \mathbf{0})$ is an equilibrium of the game $(d, X,\{\mu\},\{\alpha\})$, it is also an equilibrium of the game $(d, X,\{\mu\}, G)$ for any $d \in \mathbb{N}$.

The last step of the proof builds on a fact that was discovered by Rae and Taylor (1971). It is shown that any game $(2, X,\{\mu\},\{\alpha\})$ has an equilibrium (which, according to Proposition 2 , must be the profile $(\mathbf{0}, \mathbf{0})$ ). It should be noted that the present result is slightly different from Rae and Taylor's (1971) result, as they assumed a finite and odd number of voters. We provide our own proof of this very similar result for two reasons: first we consider infinite electorates, secondly, and more importantly, the argument in Rae and Taylor (1971) is flawed insofar as they claim that "the proof of this theorem [the existence of an equilibrium in the two-dimensional case] for any number of dimensions is immediate." Below (Example 3), we provide a counterexample to this claim (for the case with a finite and odd electorate, as well as for the case of a continuum of voters).

Without loss of generality, we only investigate deviations $y \gg \mathbf{0}$. First, we establish that for any deviation with $y_{1} \alpha_{1} \neq y_{2} \alpha_{2}$, less than half of the electorate votes for the deviator; in this case, the partisans of the deviation can either be all found above the median line $x_{2}=0$ or to the right of the median line $x_{1}=0$. (The dashed lines in Figures 3 and 4 show the set of all indifferent voters for different deviations $y$, and different shapes $\alpha$. In each case, the dashed lines separate the set of voters with a strict preference for $y$ from the set of voters with a strict preference for $\mathbf{0}$. Note, that in either case, this set is a strict subset of a "half" of the electorate, as described by the horizontal and vertical lines through $\mathbf{0 .}$.)

\section{Insert Figures 3 and 4}

Finally, for the case of $y_{1} \alpha_{1}=y_{2} \alpha_{2}$, the set of voters who prefer $y$ to $\mathbf{0}$ is a subset of the positive quadrant, while the set of voters preferring $\mathbf{0}$ to $y$ is a superset of the negative quadrant. The observation that the positive and the negative quadrant of any two-dimensional 
distribution (with median vector $\mathbf{0}$ ) must contain an equal amount of probability mass concludes the proof. (The dotted areas in Figure 5 represent the set of indifferent voters. It is important to note that the set of voters strictly preferring the deviation $y$ is a subset of the upper right quadrant, whereas the set of voters strictly preferring $\mathbf{0}$ is a superset of the lower left quadrant.)

\section{Insert Figure 5}

So $(\mathbf{0}, \mathbf{0})$ is indeed an equilibrium of the game $(2, X,\{\mu\}, G)$ when parties are uncertain about the marginal rates of substitution.

Remarks: Observe that we do not use the "common prior" assumption in this proof. The proof would also hold true if both parties where to hold different beliefs on $G$, as long as each of the parties fulfills the assumption of uncertainty about the marginal rates of substitution (two different $\alpha$ 's can be used). The only reason for the assumption of a "common prior" is that it allows us to reduce on notation. The same holds true for the results in the remainder of the paper.

Unfortunately, the result that any game $(2, X,\{\mu\},\{\alpha\})$ has an equilibrium does not extend to higher dimensions. If it did, we could apply the proof of Theorem 1 to games of any dimension, since the two-dimensionality of the game was used only to establish that any game $(2, X,\{\mu\},\{\alpha\})$ has an equilibrium. To obtain existence results for higher dimensional issue spaces, it appears that we need to introduce some uncertainty about the distribution of ideal points. But before doing so, let us conclude this section by providing an example of a three-dimensional game that does not have an equilibrium, even though the parties are uncertain about the marginal rates of substitution. Observe that this example at the same time shows that the games $(d, X,\{\mu\},\{\alpha\})$ might have no equilibria for $d>2$.

Example 3. Consider the game $(3, X,\{\mu\},\{\alpha\})$, with $X=[-1,1]^{3}$ and $\mu$ given by the following chart, where the left column denotes subspaces of $[-1,1]^{3}$ and the right column the probability mass in those subspaces. We assume that the conditional distribution in any of the subspaces $S$ is uniform. 


$\begin{array}{rcc}S & & \mu(S) \\ {[0,1]^{2} \times[-1,0)} & & .05 \\ {[0,1] \times[-1,0) \times[0,1]} & & .05 \\ {[0,1] \times[-1,0)^{2}} & .1 \\ {[-1,0)^{3}} & & .2 \\ {[-1,0)^{2} \times[0,1]} & .15 \\ {[-1,0) \times[0,1] \times[-1,0)} & .15 \\ \text { elsewhere } & 0\end{array}$

Assume that $\alpha_{1}=\alpha_{2}=\alpha_{3}=\frac{1}{3}$, that is, assume that voters care equally much about all three issues. Observe that the median vector is at the origin, $\mathbf{m}(\mu)=\mathbf{0}$, and therefore, by Proposition $2,(\mathbf{0}, \mathbf{0})$ is the only candidate for any equilibrium. But $\left(-\frac{1}{1000},-\frac{1}{1000},-\frac{1}{1000}\right)$ is a preferred deviation from this platform profile as nearly all voters with ideal points in $[-1,0)^{3}$, $[-1,0)^{2} \times[0,1],[-1,0) \times[0,1] \times[-1,0)$ and $[0,1] \times[-1,0)^{2}$ will vote for the deviator, and these sets make up $60 \%$ of the electorate. All voters in these quadrants that are sufficiently far away from the origin prefer $\left(-\frac{1}{1000},-\frac{1}{1000},-\frac{1}{1000}\right)$, as this platform is closer to their ideal point with respect to at least two dimensions. Since there are only very few voters in the immediate proximity of $\left(-\frac{1}{1000},-\frac{1}{1000},-\frac{1}{1000}\right)$ and $\mathbf{0}$, this argument concerns nearly $60 \%$ of the electorate. ${ }^{10}$

\section{$5 \quad$ Uncertainty about Electorates}

In this section, we consider the case that parties are uncertain about the distribution of ideal points $\mu$, as well as about the marginal rates of substitution. This will not only add more realism to the model, but it will also allow us to extend our prior two-dimensional existence result (Theorem 1) to higher dimensions. In particular, the main result of this section is that if the two uncertainty-averse parties do not know whether the electorate leans left or right (in a sense to be defined precisely below), and if they are also uncertain about the marginal rates of substitution, then political equilibria exist in voting games with three-dimensional issue spaces.

\footnotetext{
${ }^{10}$ The original result by Rae and Taylor (1971) only concerns finite and odd electorates. Consider the following variation of the above example as a counterexample to their claim: let the issues space and $\alpha$ be as in Example 3, but let there be 21 voters, whose ideal points are distributed as follows. 6 at $(1,1,1), 1$ at $(0,0,0),(1,1,-1)$ and $(1,-1,1)$ each, 2 at $(1,-1,-1), 4$ at $(-1,-1,-1)$ and 3 each at $(-1,-1,1)$ and $(-1,1-1)$. Observe that the median vector is at $(0,0,0)$. The deviation $\left(-\frac{1}{1000},-\frac{1}{1000},-\frac{1}{1000}\right)$ from the profile $(\mathbf{0}, \mathbf{0})$ yields an absolute majority of voters $(12)$ for the deviating party.
} 


\subsection{Characterization of equilibria}

Median vectors will again play an important role in the characterization of equilibria. In the present case, however, there is no one median vector, since parties hold multiple priors on the distribution of voter ideal points. We can characterize the set of possible equilibrium platform profiles as a function of the median vectors of all the electorate that parties take into account. Given a set $\Lambda$ of voter ideal point distributions on $\mathbb{R}^{d}$, we thus concentrate on the median set $M(\Lambda)$, which is defined as

$$
M(\Lambda):=\left\{x \in \mathbb{R}^{d}: \min _{\mu \in \Lambda} m\left(\mu_{k}\right) \leq x_{k} \leq \max _{\mu \in \Lambda} m\left(\mu_{k}\right) \text { for all } 1 \leq k \leq d\right\} .
$$

This can be thought of as the set of all platforms in-between the median platforms of the different distributions $\mu$ in $\Lambda$. The counterpart of Proposition 2 in the present context reads as follows:

Proposition 3: Let $(x, y)$ be an equilibrium of the game $(d, X, \Lambda, G)$, then $x, y \in M(\Lambda)$.

Proof: Suppose that $y \notin M(\Lambda)$, say $y_{1}<\min _{\mu \in \Lambda} m\left(\mu_{1}\right)$. Then, by the same argument as in the proof of Proposition 2, a deviation from $x$ to $x^{\prime}:=\left(\min _{\mu \in \Lambda} m\left(\mu_{1}\right), y_{2}, \ldots, y_{d}\right)$ yields for every $\psi \in \Psi(\Lambda, G)$ a vote share $\pi_{\psi}\left(x^{\prime}, y\right)>\frac{1}{2}$. Thus $\min _{\psi \in \Psi(\Lambda, G)} \pi_{\psi}\left(x^{\prime}, y\right)>\frac{1}{2}$ and as $x$ is a best response to $y$, the utility of Party $1, \min _{\psi \in \Psi(\Lambda, G)} \pi_{\psi}(x, y)$, is larger than $\frac{1}{2}$. So, in equilibrium, Party 2 only obtains a utility of $\min _{\psi \in \Psi(\Lambda, G)}\left(1-\pi_{\psi}(x, y)\right)<\frac{1}{2}$, and hence it would be strictly better off to change its platform to $x$ as $\min _{\psi \in \Psi(\Lambda, G)}\left(1-\pi_{\psi}(x, x)\right)=\frac{1}{2}$.

Remarks: (a) In passing, we note that we do not know if in equilibrium both parties need to announce the same platform. In Bade (2004), I show that if we model uncertainty aversion in a different way following Bewley (2002), equilibria with both parties announcing different platforms should always be expected to arise.

(b) It may be tempting to presume that in any equilibrium both parties must announce the median vector of some distribution in $\Lambda$. It is easy to show that this is not true. Consider the game $(2, X, \Lambda,\{\alpha\})$ where $\Lambda:=\{\lambda \nu+(1-\lambda) \phi: 0 \leq \lambda \leq 1\}$, and assume that $\operatorname{supp}(\nu)=$ $\operatorname{supp}(\phi)=[-1,2]^{2}, \nu\left([-1,1]^{2}\right)=\phi\left([0,2]^{2}\right)=.999$, and that the distribution of $\nu$ conditional on $[-1,1]^{2}$ as well as the distribution of $\phi$ conditional on $[0,2]^{2}$ are uniform. Let us finally also impose that $m\left(\nu_{1}\right)=m\left(\nu_{2}\right)$ and $m\left(\phi_{1}\right)=m\left(\phi_{2}\right)$. Then $\left(\left(\frac{1}{3}, \frac{2}{3}\right),\left(\frac{1}{3}, \frac{2}{3}\right)\right)$ is an equilibrium of this game, even though $m\left(\mu_{1}\right)=m\left(\mu_{2}\right)$ holds for all $\mu \in \Lambda$.

(c) The equilibrium platforms depend on the actual preferences of voters only insofar as beliefs of parties about the electorate are related to the actual preferences of voters. We 
consider this reasonable: the parties are picking the platforms so the outcome of the game should depend on whatever they know or believe about the electorate. The beliefs of parties depend on the information they have available; in particular a party cannot adopt a belief for strategic purposes. If we assume that the parties' information about the locations of the respective median voters is not too different from the information that is available to researchers, then the present theory can be used to predict equilibrium platforms.

(d) The assumption of common belief sets is not necessary. However, observe that the belief sets need to be close enough in the sense that in equilibrium both parties must locate in $M\left(\Lambda_{1}\right)$ as well as in $M\left(\Lambda_{2}\right)$. They can do so if and only if the intersection between these two sets is not empty. If the intersection is empty, the beliefs of parties diverge so far as to allow for strategy profiles in which each party is sure it will win the election. Billot et al. (2000) develop a notion of "Sharing Beliefs" that generalizes the idea of common priors to environments with multiple agents that all have maxmin expected utilities following Gilboa and Schmeidler (1989). If the criterion mentioned in Billot et. al. (2000) is satisfied in the present context, then the intersection of $M\left(\Lambda_{1}\right)$ and $M\left(\Lambda_{2}\right)$ must be non-empty.

\subsection{The Existence of Equilibrium}

To state sufficient conditions for the existence of equilibria, we have to introduce some more concepts. Given a distribution of $\mu$, let us agree to call a voter a leftist if her ideal point lies below the median vector with respect to all issues. That is, a voter with ideal point $a \in X$ is a leftist (rightist) iff $a_{i}<m\left(\mu_{i}\right)\left(a_{i}>m\left(\mu_{i}\right)\right.$, respectively) for all issues $i$. Given some distribution $\mu$ of voter ideal points, we denote the set of all leftists and rightists by $A_{l}^{\mu}$ and $A_{r}^{\mu}$ respectively. A distribution $\mu$ is called left-leaning (right-leaning) if $\mu\left(A_{l}^{\mu}\right) \geq \mu\left(A_{r}^{\mu}\right)\left(\mu\left(A_{r}^{\mu}\right) \leq \mu\left(A_{l}^{\mu}\right)\right.$ respectively). Finally, a distribution $\mu$ with equally many leftists and rightists $\left(\mu\left(A_{l}^{\mu}\right)=\mu\left(A_{r}^{\mu}\right)\right)$ is called balanced. Observe that any balanced distribution is left- and right- leaning at the same time. The condition of balance is much weaker than the condition that a generalized median exists: while the former imposes one equality on the distribution, the latter imposes a continuum of equalities, as in this case each hyperplane through the generalized median must cut the electorate into half spaces with an equal amount of voter ideal points in them. We say a party is uncertain as to whether the electorate leans to the left or to the right if there exists a left-leaning and a rightleaning distribution in the set of party beliefs on the distribution of voter ideal points. Just as with the definition of uncertainty about the marginal rates of substitution, the present definition covers one case of certainty. Parties who hold a singleton belief $\Lambda=\{\mu\}$, which is balanced, satisfy the definition of uncertainty as to whether the electorate leans left or right.

Theorem 2: Let $(3, X, \Lambda, G)$ be a game where parties are uncertain about the marginal 
rates of substitution and uncertain as to whether the electorate leans to the left or to the right. Then $(3, X, \Lambda, G)$ has a political equilibrium.

Theorem 1 arises as a special case of Theorem 2 due to the following two observations: first of all, two-dimensional games are embedded in three-dimensional games (by simply adding a dummy dimension on which all players have the same ideal point $a_{3}$ and attitude $g_{3}$ ). Secondly, any two-dimensional distribution is balanced, since in the two-dimensional case $\mu\left(A_{l}^{\mu}\right)$ can be calculated as $1-\mu\left(\left\{a: a_{1}>m\left(\mu_{1}\right)\right\}\right)-\mu\left(\left\{a: a_{2}>m\left(\mu_{2}\right)\right\}\right)+\mu\left(A_{r}^{\mu}\right)=\mu\left(A_{r}^{\mu}\right)$, where $\left\{a: a_{1}>m\left(\mu_{1}\right)\right\}$ and $\left\{a: a_{2}>m\left(\mu_{2}\right)\right\}$ denote the half spaces to the right of the median line $m\left(\mu_{1}\right)$ and above the median line $m\left(\mu_{2}\right)$, respectively. Again in analogy to Theorem 1, Theorem 2 covers one case in which parties are expected utility maximizers. According to the theorem, equilibria exist in the knife-edge case of $(3, X,\{\mu\}, \alpha)$ for any balanced $\mu$ and any diamond-shaped indifference curves $\alpha$. The proof of Theorem 2 is reminiscent of the proof of Theorem 1 . We showed already that under the assumption that parties are uncertain about the marginal rates of substitution, there exists an $\alpha \in \mathbb{R}_{++}^{d}$ such that the equilibrium set of $(3, X,\{\mu\},\{\alpha\})$ is a subset of the equilibrium set of $(3, X,\{\mu\}, G)$ for any fixed $\mu$. If we can find a $\mu \in \Lambda$ such that $(3, X,\{\mu\},\{\alpha\})$ has an equilibrium, the proof is complete, since we know that any equilibrium for some game of electoral competition is also an equilibrium in a game with larger belief sets (Lemma 1). The main difficulty lies in the fact that $(3, X,\{\mu\},\{\alpha\})$ need not have an equilibrium (see Example 3). Yet, in the appendix we generalize Rae and Taylor (1971) and show that $(3, X,\{\mu\},\{\alpha\})$ has an equilibrium if and only if $\mu$ is balanced. Given our assumption that the parties are uncertain as to whether the electorate is left- or right-leaning we can show that there exists some balanced $\mu^{*}$ in $\Lambda$, and we thus obtain that $\left(\mathbf{m}\left(\mu^{*}\right), \mathbf{m}\left(\mu^{*}\right)\right)$ is an equilibrium of $(3, X, \Lambda, G)$.

The following example shows that the conditions given in Theorem 2 are sufficient, but not necessary, for the existence of an equilibrium.

Example 4: Let $\left(3,[-1,1]^{3}, \Lambda, G\right)$ be a game with $\Lambda$ the set of all convex combinations of the voter ideal point distributions $\mu^{1}, \ldots, \mu^{8}$. Assume that all of these distributions $\mu^{i}$ are right-leaning. Assume furthermore that, for each of the eight octants around $\mathbf{0}$, there is a distribution $\mu^{i}$ amongst $\mu^{1}, \ldots, \mu^{8}$ such that the interior of that octant contains at least .6 of the probability mass according to $\mu^{i}$. (Observe that we do not require $\mathbf{0}$ to be the median vector of any one of these distributions of voter ideal points.) We claim that $\mathbf{0}$ is an equilibrium of that game. Observe that, by our assumption that all voters' preferences are single-peaked, for any deviation $y$ from $\mathbf{0}$, there exists some octant such that all voters in that octant are voting for $\mathbf{0}$. Now, by our construction of the set $\Lambda$ there exists a $\mu$ in it such that at least .6 of all voters prefer $\mathbf{0}$ to $y$ according to that $\mu$. Consequently, $(\mathbf{0}, \mathbf{0})$ is an 
equilibrium, even though by construction all distributions $\mu \in \Lambda$ are right-leaning. Observe also that we did not specify $G$ to obtain this result, $G$ might even be a singleton.

Roughly speaking, Theorem 2 establishes that any three-dimensional game played amongst parties that are neither certain about the voters' marginal rates of substitution nor about the leanings of the electorate has an equilibrium. Example 4 shows that this amount of uncertainty is sufficient, but not necessary, for the existence of equilibria. However, from Proposition 3 we know that in any equilibrium, whether the sufficient conditions are fulfilled or not, both parties have to announce a policy from the median set. This means that with respect to every issue $i$, parties announce the ideal of a voter who is the median voter of the marginal distribution $\mu_{i}$ of some $\mu \in \Lambda$.

\section{$6 \quad$ Higher-Dimensional Issue Spaces}

In the introduction, we argued that empirical studies on the dimensionality of policy spaces usually find that very few dimensions suffice to explain voting behavior. Consequently, the extension of the present results to policy spaces with more than three dimensions is mainly of technical interest. For completeness, we develop a variation of Theorem 3 that applies to higher-dimensional policy spaces. To this end, we need one further bit of notation.

Let us define the function sgn: $\mathbb{R}^{d} \rightarrow\{1,0,-1\}^{d}$ by $\operatorname{sgn}(x)=\left(\operatorname{sgn}\left(x_{1}\right), \ldots, \operatorname{sgn}\left(x_{d}\right)\right)$, and let

$$
A_{f}^{\mu}:=\{x \mid \operatorname{sgn}(x-m(\mu))=f\} \text { for all } f \in\{1,-1\}^{d} .
$$

The expressions $A_{f}^{\mu}$ describe the "quadrants" around the median vector of a distribution. We call a distribution $\mu \in \mathbb{P}(X)$ equilibrated, if there is the same amount of probability mass in each pair of opposing quadrants, that is, $\mu\left(A_{f}^{\mu}\right)=\mu\left(A_{-f}^{\mu}\right)$ for all $f \in\{1,-1\}^{d}$. Note that any balanced three-dimensional distribution and any two-dimensional distribution is equilibrated. Also note that the condition is less restrictive as the condition that a generalized median exists: while for a distribution to be equilibrated less than $2^{d}$ equations have to whole, the existence of a generalized median requires a continuum of equations to hold.

Theorem 3: Let $d \in \mathbb{N}$ and let $(d, X, \Lambda, G)$ be a game such that parties are uncertain about the marginal rates of substitution and that there exists an equilibrated $\mu$ in $\Lambda$, then $(d, X, \Lambda, G)$ has an equilibrium.

In this result, we require that there exists some equilibrated $\mu$ in $\Lambda$, whereas Theorem 2 requires that parties do not know whether the electorate leans to the left or to the right. Within three-dimensional voting games, these conditions coincide. Consequently, Theorem 3 generalizes Theorem 2 (and thereby Theorem 1) to the context of a $d$-dimensional issue 
space. The proof of Theorem 3 is analogous to that of Theorem 2. In fact, a crucial step in the proof of Theorem 2 was to establish the existence of an equilibrated $\mu$ in $\Lambda$ under the conditions of that theorem. In Theorem 3, we simply impose the existence of such a distribution as a hypothesis. From then on, both proofs are identical.

The condition that there exists an equilibrated $\mu$ in $\Lambda$ is, admittedly, not very intuitive. However, it may well be the case that the parties' uncertainty about electorates increases with the dimensionality of the issue space. Perhaps this uncertainty about the distribution of ideal points may indeed be large enough that some equilibrated distributions could be considered a plausible assumption on the voter ideal point distribution by the agenda setters of the parties. At any rate, Theorem 3 (like Theorem 2) provides only sufficient conditions. Example 4 demonstrates that there remains room for weaker conditions that would ensure the existence of equilibrium.

\section{Conclusion}

This paper provides an example for Ordeshook's claim that incorporation of an assumption that "candidates are uncertain about voters' exact preferences" can mitigate problems of equilibrium non-existence in games of electoral competition. Given that parties are sufficiently uncertain, equilibria exist in two-party games of electoral competition when multiple issues are at stake. To be precise, it was shown that any game of electoral competition with a policy space of at most three dimensions has an equilibrium if parties are uncertain about the marginal rates of substitution of voters and whether the electorate leans left or right. What is more, in these equilibria both parties announce issue by issue the policy preferred by the "median voter" that is relevant for the issue under consideration. So this theory can be used to justify the common practice to look at separate issues when modeling democratic processes.

It has to be said that the proofs in the paper rely on the assumption that the sets of party beliefs contain some belief according to which an equilibrium exists. Since according to these beliefs the games would also have equilibria if parties were not uncertainty-averse, one might ask: why introduce uncertainty aversion, when one could also obtain equilibrium existence by positing that parties hold exactly such beliefs about the electorate? The reason is that the assumption that parties would hold precisely the belief according to which an equilibrium exists seems overly strong. Why would parties believe that the indifference curves of voters are diamond shaped and that there are exactly as many leftists and rightists in the electorate? Conversely, the assumption that this is just one of the many beliefs that parties entertain seems much less demanding. The upshot of uncertainty aversion is that we do not need to impose some knife-edge condition on party beliefs to obtain equilibrium; 
instead, it is sufficient to establish that parties consider the cases in which an electorate leans left or right and that parties are uncertain about the marginal rates of substitution to obtain equilibria.

The conditions for the existence of equilibrium given in the theorems of this paper are sufficient, but not necessary. It is hoped that in the future some weaker conditions for the existence of equilibria will be established. A promising venue could be to assume Euclidean preferences and to restrict the set of permissible voter ideal point distributions and then ask what amount of uncertainty is sufficient to establish that equilibria exist. In particular, we hope to show that under the assumption that the society is not polarized (following Caplin and Nalebuff (1988)), a small amount of uncertainty on the voter ideal point distribution is sufficient to establish the existence of equilibria in $d$ dimensional games.

Another extension of this research could be to apply the same model of uncertainty aversion to solve different but related non-existence problems in political economy. Some models of ideologically motivated parties, for example, are plagued by similar non-existence problems as the Downs model. A main challenge in extending the present framework to such models lies in defining the utilities of parties. Electoral competition with more than 2 parties could be another area of application. Platform-positioning models with more than two parties generally lack equilibria. Uncertainty aversion could be one of the modelling approaches to trim the model's incentives to deviate down to a more realistic lower level.

Furthermore, in a companion paper (Bade 2004), we show that with a different approach towards modelling uncertainty-averse actors (following Bewley 2002), there might be equilibria in which office-motivated parties announce different platforms. Our explanation for platform divergence does not need any ad hoc assumptions on the ideological motivation or parties; the driving force of this result are non-convexities in the preferences of voters.

\section{Appendix}

Proof of Proposition 1: We will need the following intermediate result.

Define $\psi^{\prime}: \mathcal{B}(X \times H) \rightarrow[0,1]$ by $\psi^{\prime}(A):=\int_{\mathbb{P}^{*}(X \times H)} \psi(A) p(d \psi)$, for an arbitrarily fixed $p \in \mathcal{P}$. This is well-defined as $\psi \mapsto \psi(A)$ is a Borel-measurable function, which can be shown using Theorem 14.13 in Aliprantis and Border (1999), letting $f=1_{A}$. Moreover, it is obvious that $\psi^{\prime}(\varnothing)=0$ and $\psi^{\prime}(X \times H)=1$. To conclude that $\psi^{\prime}$ is a Borel probability measure on $X \times H$, let $\left\{A_{1}, A_{2}, \ldots\right\}$ be a countable set of mutually disjoint Borel sets in $X \times H$, and notice that

$$
\psi^{\prime}\left({ }_{i=1}^{\infty} A_{i}\right)=\int_{\mathbb{P}^{*}(X \times H)} \psi\left({ }_{i=1}^{\infty} A_{i}\right) p(d \psi)=\sum_{i=1}^{\infty} \int_{\mathbb{P}^{*}(X \times H)} \psi\left(A_{i}\right) p(d \psi)=\sum_{i=1}^{\infty} \psi^{\prime}\left(A_{i}\right) .
$$


by the monotone convergence theorem. Since it is also easy to show that $\psi$ is non-atomic and $\psi_{a}$ has full support, it follows that $\Psi \subseteq \mathbb{P}^{*}(X \times H)$.Moreover, for any $p, q \in \mathcal{P}$ and any $\lambda \in[0,1]$, we have

$$
\lambda \int_{\mathbb{P}^{*}(X \times H)} \psi p(d \psi)+(1-\lambda) \int_{\mathbb{P}^{*}(X \times H)} \psi q(d \psi)=\int_{\mathbb{P}^{*}(X \times H)} \psi(\lambda p+(1-\lambda) q)(d \psi),
$$

so the convexity of $\Psi$ follows from that of $\mathcal{P}$. Finally, for any $(x, y) \in X^{2}$, by the definition of $\pi_{\psi}$,

$$
\begin{aligned}
\int_{\mathbb{P}^{*}(X \times H)} \pi_{\psi}(x, y) p(d(\psi)) & =\int_{\mathbb{P}^{*}(X \times H)}\left(\psi(T(x, y))+\frac{1}{2} \psi(I(x, y))\right) p(d \psi) \\
& =\psi^{\prime}(T(x, y))+\frac{1}{2} \psi^{\prime}(I(x, y))=\pi_{\psi^{\prime}}(x, y) .
\end{aligned}
$$

This completes the proof of Proposition 1.

\section{Proof of Theorem 1:}

Lemma 2 (Rae and Taylor, 1971): Any game of the form $(2, X,\{\mu\},\{\alpha\})$ has an equilibrium.

Proof: Suppose some profitable deviation $y \gg \mathbf{0}$ existed (remember that we normalized $\mathbf{m}(\mu)=\mathbf{0})$. Let $A$ be the set of voters that are indifferent between $\mathbf{0}$ and $y$. Given that every voter has the same shape $\alpha$ we have that all voters $a^{\prime} \notin A$ for which there exists an $a \in A$ such that $a^{\prime} \ll a$ strictly prefer 0 to $y$. If $A \cap\left\{a \mid a_{1}=0\right\}=\varnothing$ or $A \cap\left\{a \mid a_{2}=0\right\}=\varnothing$ then since the $A$ is a connected set either all voters in $\left\{a \mid a_{1} \leq 0\right\}$ or all voters in $\left\{a \mid a_{2} \leq 0\right\}$ will prefer 0 to $y$. Figures 1 and 2 give two examples for these two cases; the dashed lines represent the set $A$, observe that in either there is one axis that does not intersect with the dashed lines. Since $(\mathbf{0}, \mathbf{0})$ is the median vector, we have $\mu\left(\left\{a \mid a_{1} \leq 0\right\}\right) \geq \frac{1}{2}$ and $\mu\left(\left\{a \mid a_{2} \leq 0\right\}\right) \geq \frac{1}{2}$, so in either case at least half the electorate votes for $\mathbf{0}$ and therefore such a deviation to $y$ cannot raise the deviating party's vote share.

Let us now consider the remaining case in which $A \cap\left\{a \mid a_{1}=0\right\} \neq \varnothing$ and $A \cap\left\{a \mid a_{2}=\right.$ $0\} \neq \varnothing$. This only holds for deviations $y$ such that $y_{1} \alpha_{1}=y_{2} \alpha_{2}$. In this case, all voters in $\left\{a \mid a_{1} \leq 0\right.$ and $\left.a_{2} \geq y_{2}\right\}$ and in $\left\{a \mid a_{1} \geq y_{1}\right.$ and $\left.a_{2} \leq 0\right\}$ are indifferent between $\mathbf{0}$ and $y$. Since we assume that all indifferent voters vote for either platform with equal probability, we only need to look at the voters that strictly prefer one platform to the other. The set of voters strictly preferring $y$ to $\mathbf{0}$ is a subset of $\left\{a \mid a_{1}>0, a_{2}>0\right\}$, whereas the set of voters strictly prefer $\mathbf{0}$ to $y$ is a superset of $\left\{a \mid a_{1}<0, a_{2}<0\right\}$. But since $(\mathbf{0}, \mathbf{0})$ is the median vector of the non-atomic $\mu$ we have $\mu\left(\left\{a \mid a_{1}>0, a_{2}>0\right\}\right)=\mu\left(\left\{a \mid a_{1}<0, a_{2}<0\right\}\right)$. Consequently, 
it cannot be that such a deviation increases the vote share. But by the same arguments no other deviation $y$ raises the vote share to the deviating party and $(\mathbf{0 , 0})$ is a political equilibrium.

Lemma 3: Let $d \in \mathbb{N}$, and let $(d, X,\{\mu\}, G)$ be a game in which parties are uncertain about the marginal rates of substitution. Then, for all $y \in X$, there exists an electorate $\psi^{y}$ in $\Psi(\{\mu\}, G)$ such that $\pi_{\psi^{y}}(y, \mathbf{0}) \leq \pi_{\mu \times \alpha}(y, \mathbf{0})$.

Proof: Fix an arbitrary $y \in X$. Since parties are uncertain about the marginal rates of substitution, there exists an $\alpha \in \mathbb{R}_{++}^{d}$ such that in all $G_{i}$ there are some differentiable functions $h_{i}$ and $k_{i}$ with $h_{i}^{\prime} \geq \alpha_{i} \geq k_{i}^{\prime}$. Define the map $a \longmapsto\left(g_{1}^{a}, \ldots, g_{d}^{a}\right)=: g^{a}$ by

$$
g_{i}^{a}:=\left\{\begin{array}{ll}
h_{i}, & \text { if }\left|y_{i}-a_{i}\right| \geq\left|a_{i}\right| \\
k_{i}, & \text { otherwise }
\end{array}, i=1, \ldots, d\right.
$$

We begin by showing that $u^{\alpha}(\mathbf{0}, a)>u^{\alpha}(y, a)$ implies $u^{g^{a}}(\mathbf{0}, a)>u^{g^{a}}(y, a)$ for all $a$. Observe, first of all, that $u^{\alpha}(\mathbf{0}, a)>u^{\alpha}(y, a)$ implies

$$
\sum_{i \in H} \alpha_{i}\left(\left|y_{i}-a_{i}\right|-\left|a_{i}\right|\right)>\sum_{i \notin H} \alpha_{i}\left(\left|a_{i}\right|-\left|y_{i}-a_{i}\right|\right)
$$

where $H:=\left\{i \in\{1, \ldots, d\}|| y_{i}-a_{i}|\geq| a_{i} \mid\right\}$.Then, since $h_{i}^{\prime} \geq \alpha_{i} \geq k_{i}^{\prime}$,

$$
\begin{cases}h_{i}\left(\left|y_{i}-a_{i}\right|\right)-h_{i}\left(\left|a_{i}\right|\right) \geq \alpha_{i}\left|y_{i}-a_{i}\right|-\alpha_{i}\left|a_{i}\right|, & \text { if }\left|y_{i}-a_{i}\right| \geq\left|a_{i}\right| \\ \alpha_{i}\left|a_{i}\right|-\alpha_{i}\left|y_{i}-a_{i}\right| \geq k_{i}\left(\left|a_{i}\right|\right)-k_{i}\left(\left|y_{i}-a_{i}\right|\right), & \text { otherwise. }\end{cases}
$$

Combining these inequalities with the definition of $g^{a}$, we obtain

$$
\sum_{i=1}^{d}\left(g_{i}^{a}\left(\left|y_{i}-a_{i}\right|\right)-g_{i}^{a}\left(\left|a_{i}\right|\right)\right)>0
$$

that is, $u^{g^{a}}(\mathbf{0}, a)>u^{g^{a}}(y, a)$. One can show similarly that $u^{\alpha}(\mathbf{0}, a)=u^{\alpha}(y, a)$ implies $u^{g^{a}}(\mathbf{0}, a) \geq u^{g^{a}}(y, a)$ for all $a$.

Next, define an electorate $\psi^{y}$ such that on the one hand the conditional distribution of shapes given a voter ideal point $a$ can be described by the map $a \mapsto g^{a}$, that is, we assume that $\psi\left(g^{a} \mid a\right)=1$ for all $a$ and that, on the other hand, the voter ideal point distribution $\psi_{a}$ equals $\mu$. Since the map $a \mapsto g^{a}$ is measurable, $\psi^{y}$ is well-defined. Moreover, by construction, 
we have that $\psi^{y} \in \Psi(\{\mu\}, G)$. The construction of the map $a \longmapsto g^{a}$ also implies:

$$
\begin{aligned}
\pi_{\psi^{y}}(y, \mathbf{0}) & =1-\psi^{y}\left\{(a, g) \mid u^{g}(\mathbf{0}, a)>u^{g}(y, a)\right\}-\frac{1}{2} \psi^{y}\left\{(a, g) \mid u^{g}(\mathbf{0}, a)=u^{g}(y, a)\right\} \\
& =1-\mu\left\{a \mid u^{g^{a}}(\mathbf{0}, a)>u^{g^{a}}(y, a)\right\}-\frac{1}{2} \mu\left\{a \mid u^{g^{a}}(\mathbf{0}, a)=u^{g^{a}}(y, a)\right\} \\
& \leq 1-\mu\left\{a \mid u^{\alpha}(\mathbf{0}, a)>u^{\alpha}(y, a)\right\}-\frac{1}{2} \mu\left\{a \mid u^{\alpha}(\mathbf{0}, a)=u^{\alpha}(y, a)\right\} \\
& \left.=1-\left(\mu \times \delta_{\alpha}\right)\left\{(a, g) \mid u^{g}(\mathbf{0}, a)>u^{g}(y, a)\right\}\right)-\frac{1}{2}(\mu \times \alpha)\left\{(a, g) \mid u^{g}(\mathbf{0}, a)=u^{g}(y, a)\right\} \\
& =\pi_{\mu \times \alpha}(y, \mathbf{0}),
\end{aligned}
$$

and the proof is complete.

Proof of Theorem 1: By Proposition 1, we know that the only candidate for an equilibrium is $(\mathbf{0}, \mathbf{0})$. The payoff to a party that deviates to $y$ is $\min _{\psi \in \Psi(\{\mu\}, G)} \pi_{\psi}(y, \mathbf{0})$. By Lemma $3, \pi_{\mu \times \alpha}(y, \mathbf{0})$ is an upper bound on this payoff. Finally, by Lemma 2, according to the electorate $\mu \times \alpha$ no profitable deviation form $(\mathbf{0}, \mathbf{0})$ exists. Thus,

$$
\min _{\psi \in \Psi(\{\mu\}, G)} \pi_{\psi}(y, \mathbf{0}) \leq \pi_{\psi^{y}}(y, \mathbf{0}) \leq \pi_{\mu \times \alpha}(y, \mathbf{0}) \leq \frac{1}{2}=\min _{\psi \in \Psi(\{\mu\}, G)} \pi_{\psi}(\mathbf{0}, \mathbf{0})
$$

where $\psi^{y}$ is the electorate constructed in Lemma 3. So there is no profitable deviation for either party, and $(\mathbf{0}, \mathbf{0})$ is an equilibrium of $(2, X,\{\mu\}, G)$.

The following Lemma 4 is a major building block of the proof of Theorem 2 .

Lemma 4: A game of the form $(3, X,\{\mu\},\{\alpha\})$ has an equilibrium if and only if $\mu$ is balanced.

Proof: We define the function sgn: $\mathbb{R}^{3} \rightarrow\{1,0,-1\}^{3}$ by $\operatorname{sgn}(x)=\left(\operatorname{sgn}\left(x_{1}\right), \operatorname{sgn}\left(x_{2}\right), \operatorname{sgn}\left(x_{d}\right)\right)$, and let $A_{f}^{\mu}:=\{x \mid \operatorname{sgn}(x-m(\mu))=f\}$ for all $f \in\{1,-1\}^{3}$. As Lemma 4 covers games with certainty, we can, for ease of exposition, revert to the normalization $\mathbf{m}(\mu)=\mathbf{0}$. We therefore also drop $\mu$ from the notation of these sets and now write $A_{l}, A_{r}$ and $A_{f}$.

We first show that $\mu\left(A_{l}\right)=\mu\left(A_{r}\right)$ if and only if $\mu\left(A_{f}\right)=\mu\left(A_{-f}\right)$ for all $f$, then we show that this condition is necessary and sufficient for $(\mathbf{0}, \mathbf{0})$ being an equilibrium.

Let $\mu\left(A_{l}\right)=\mu\left(A_{r}\right)$. Define 4 variables $D_{f}:=\mu\left(A_{f}\right)-\mu\left(A_{-f}\right)$ for all $f$ with $f_{1}=1$. Since $\mathbf{0}$ is the median vector of $\mu$ we have that $D_{f}=0$ for all $i=1,2,3$. Given $D_{(1,1,1)}=$ $\mu\left(A_{r}\right)-\mu\left(A_{l}\right)=0$ this reduces to a system of 3 linearly independent equations in 3 unknowns, the only solution is $D_{f}=\mu\left(A_{f}\right)-\mu\left(A_{-f}\right)=0$ for all $f$.

Given $\mu\left(A_{f}\right)=\mu\left(A_{-f}\right)$ for all $f$ we show that for any deviation from $(\mathbf{0}, \mathbf{0})$ the party remaining at $\mathbf{0}$ gets at least half the vote share. First, we derive a condition under which 
all voters in some $A_{f}$ vote for $\mathbf{0}$. Then we use this condition to show that given the choice between the platforms $y \neq \mathbf{0}$ and $\mathbf{0}$, for any $f$, either all voters in $A_{f}$ or all voters in $A_{-f}$ vote for $\mathbf{0}$ or no voter in either $A_{f}$ or $A_{-f}$ strictly prefers $y$ to $\mathbf{0}$.

All voters in $A_{f}$ vote for 0 if for all $a \in A_{f}$ the utility from platform $0:-\sum_{i=1}^{3} \alpha_{i}\left|a_{i}\right|$ is larger than the utility from the other platform: $-\sum_{i=1}^{3} \alpha_{i}\left|y_{i}-a_{i}\right|$. So all voters in $A_{f}$ strictly prefer $\mathbf{0}$ to $y$ if

$$
\sup _{a \in A_{f}}\left(\sum_{i=1}^{3} \alpha_{i}\left|a_{i}\right|-\sum_{i=1}^{3} \alpha_{i}\left|y_{i}-a_{i}\right|\right)<0 \text {. }
$$

Considering all possible cases, we can solve for the absolute value of $\left|a_{i}\right|$ and $\left|y_{i}-a_{i}\right|$ and receive

$$
\begin{gathered}
\sup _{a \in A_{f}}\left(\sum_{y_{i} \geq 0 \geq a_{i}}\left(-\alpha_{i} y_{i}\right)+\sum_{0 \leq a_{i} \leq y_{i}}\left(2 \alpha_{i} a_{i}-\alpha_{i} y_{i}\right)+\sum_{0 \leq y_{i} \leq a_{i}} \alpha_{i} y_{i}+\right. \\
\left.+\sum_{y_{i}<0<a_{i}} \alpha_{i} y_{i}+\sum_{y_{i} \leq a_{i}<0}\left(-2 \alpha_{i} a_{i}+\alpha_{i} y_{i}\right)+\sum_{a_{i}<y_{i} \leq 0}-\alpha_{i} y_{i}\right)=\sum_{i=1}^{3} f_{i} \alpha_{i} y_{i} .
\end{gathered}
$$

So if this expression is negative the proof is complete as all voters in $A_{f}$ vote for $\mathbf{0}$. If this expression is positive we have

$$
-\sum_{i=1}^{3} f_{i} \alpha_{i} y_{i}=\sum_{i=1}^{3}-f_{i} \alpha_{i} y_{i}<0
$$

and therefore all voters in $A_{-f}$ vote for $\mathbf{0}$. Finally, if $\sum_{i=1}^{d} f_{i} \alpha_{i} y_{i}=0$ then

$$
\sup _{a \in A_{f}}\left(\sum_{i=1}^{3} \alpha_{i}\left|a_{i}\right|-\sum_{i=1}^{3} \alpha_{i}\left|y_{i}-a_{i}\right|\right)=\sup _{a \in A_{-f}}\left(\sum_{i=1}^{3} \alpha_{i}\left|a_{i}\right|-\sum_{i=1}^{3} \alpha_{i}\left|y_{i}-a_{i}\right|\right)=0 \text {. }
$$

and consequently no voter in either $A_{f}$ or $A_{-f}$ strictly prefers platform $y$.

Given that $\mu\left(A_{-f}\right)=\mu\left(A_{f}\right)$ for all $f$ we can now show that $\sum_{f_{1}=1} \mu\left(A_{f}\right)$ represents a lower bound on $\pi(\mathbf{0}, y)$. This is so since, on the one hand, for any $f$ with $f_{1}=1$ and any deviation $y$ either all voters with ideal point in $A_{f}$ or all voters with ideal point in $A_{-f}$ or one half of the voters with ideal point in $A_{f} \cup A_{-f}$ vote for $\mathbf{0}$, and, on the other hand, $\mu\left(A_{-f}\right)=\mu\left(A_{f}\right)$ for all $f$. Finally, since $\mathbf{0}$ is the median vector of $\mu$ we know that $\sum_{f_{1}=1} \mu\left(A_{f}\right)=\frac{1}{2}$ and we have $\pi_{\mu}(\mathbf{0}, y) \geq \frac{1}{2}$ and therefore no deviation from $(\mathbf{0}, \mathbf{0})$ that raises the vote share of the deviating party exists.

Now suppose $\mu$ were not balanced, that is, assume $\mu\left(A_{l}\right)=p$ and $\mu\left(A_{r}\right)=q$ with $p>q$. Let the values of all $\mu\left(A_{f}\right)$ be given by the following chart: 


\begin{tabular}{rc}
\multicolumn{1}{c}{$f$} & $\mu\left(A_{f}\right)$ \\
\cline { 1 - 1 }$(1,1,1)$ & $q$ \\
$(1,1,-1)$ & $s$ \\
$(1,-1,1)$ & $\frac{1}{2}-s-r-q$ \\
$(-1,-1)$ & $s+(q-p)$ \\
$(-1,-1,1)$ & $r+(q-p)$ \\
$(-1,1,-1)$ & \\
$(-1,1,1)$ & $\frac{1}{2}-s-r-q-(q-p)$
\end{tabular}

When a deviator plays $\lambda\left(\frac{1}{\alpha_{1}}, \frac{1}{\alpha_{2}}, \frac{1}{\alpha_{3}}\right)$ with $\lambda>0$ against $\mathbf{0}$, then all voters in $A_{l}, A_{(1,-1,-1)}, A_{(-1,-1,1)}$ and $A_{(-1,1,-1)}$ are voting for $\mathbf{0}$. In the limit for $\lambda \rightarrow 0$ only these voters will vote for $\mathbf{0}$. So in the limit the vote share of the remaining party is $\frac{1}{2}+q-p$. Since the vote share of the remaining party decreases continuously with $\lambda$, there exists some $\lambda^{*}>0$ such that $\pi\left(\lambda^{*}\left(\frac{1}{\alpha_{1}}, \frac{1}{\alpha_{2}}, \frac{1}{\alpha_{3}}\right), \mathbf{0}\right)>\frac{1}{2}$ and $(\mathbf{0}, \mathbf{0})$ cannot be an equilibrium.

Before proceeding with the proof of Theorem 2, let us remark that Lemma 4 generalizes Lemma 3 as any two-dimensional distribution of voter ideal points is balanced. Secondly, observe that balance of $\mu$ does not imply $\mu\left(A_{f}\right)=\mu\left(A_{-f}\right)$ for all $f$, for higher-dimensional issue spaces.

Proof of Theorem 2: We start by showing that there exists a balanced $\mu$ in $\Lambda$. By our assumption that the parties are uncertain as to whether the electorate leans to the left or right, there exists a left-leaning $\mu_{l}$ and a right-leaning $\mu_{r}$ in $\Lambda$. For all $\lambda \in[0,1]$ define $\mu_{\lambda}=\lambda \mu_{l}+(1-\lambda) \mu_{r}$. Define

$$
\begin{aligned}
f & : \quad[0,1] \rightarrow[-1,1] \\
f(\lambda) & =\mu_{\lambda}\left(A_{l}^{\mu_{\lambda}}\right)-\mu_{\lambda}\left(A_{r}^{\mu_{\lambda}}\right)
\end{aligned}
$$

a continuous function. Clearly: $f(1)>0$ and $f(0)<0$ so there exists some $\lambda^{b} \in(0,1)$ such that $f\left(\lambda^{b}\right)=0$. Observe that $\mu_{\lambda^{b}}$ is balanced. Since $\Lambda$ convex, we also have that $\mu_{\lambda^{b}} \in \Lambda$.

By the same argument as forwarded in the proof of Theorem 1, we know that $\left(\mathbf{m}\left(\mu_{\lambda^{b}}\right), \mathbf{m}\left(\mu_{\lambda^{b}}\right)\right)$ is an equilibrium of $\left(3, X,\left\{\mu_{\lambda^{b}}\right\}, G\right)$. We can conclude by Lemma 1 that it is also an equilibrium of $(3, X, \Lambda, G)$.

Proof of Theorem 3: Following the proof of Theorem 2, observe that once we had established that $\mu\left(A_{f}\right)=\mu\left(A_{-f}\right)$ for all $f$ in Lemma 3 , we made no more use of either balance or three-dimensionality in the proofs of Lemma 3. So we note the following Lemma 5 in passing: 
Lemma 5: Take an d-dimensional game $(d, X,\{\mu\},\{\alpha\})$ with $\mathbf{m}(\mu)=\mathbf{0}$. This game has an equilibrium if $\mu\left(A_{f}\right)=\mu\left(A_{-f}\right)$ for all $f$.

The proof of Theorem 3 proceeds like that of Theorem 2, replacing Lemma 4 by Lemma 5.

\section{References}

Aliprantis, C., Border, K., 1999. Infinite Dimensional Analysis, A Hitchiker's Guide. 2nd ed.. Springer, Berlin.

Ashworth, S., 2007. Targeted Campaigns with Ambiguity Averse Voters. mimeo, Princeton University.

Bade, S., 2004. Divergent Platforms. mimeo, New York University.

Banks, J. and Duggan J. 2006. Probabilistic Voting in the Spatial Model of Elections: The Theory of Office-Motivated Candidates. in Social Choice and Strategic Decisions: Essays in Honor of Jeffrey S. Banks, David Austen-Smith and John Duggan, eds., New York: SpringerVerlag.

Barbera, S., Gul, F., Stachetti, E., 1993. Generalized Median Voter Schemes and Committees. Journal of Economic Theory 61, 262-289.

Berliant, M., Konishi, H., 2005. Salience: Agenda Choices by Competing Candidates, Public Choice 125, 129-149.

Besley, T., Coate, S., 1997. An Economic Model of Representative Democracy, Quarterly Journal of Economics 112, 85-114.

Bewley, T., 2002. Knightian Utility Theory: Part 1. Decision is Economics and Finance $25,79-110$.

Billot, A., Chateauneuf, A., Gilboa, I., Tallon, J-M., 2000. Sharing Beliefs: Between Agreeing and Disagreeing. Econometrica 68, 685-694.

Black, D., 1948. On the Rationale of Group Decision-Making. Journal of Political Economy 56, 2334.

Camerer, C., Weber, M., 1992. Recent Developments in Modeling Preferences: Uncertainty and Ambiguity. Journal of Risk and Uncertainty 5, 325-370. 
Caplin, A., Nalebuff, B., 1988. On 64\% Majority Rule. Econometrica 56, 787-814.

Coughlin, P., 1992. Probabilistic Voting Theory. Cambridge University Press.

Davis, O., de Groot M., Hinich, M., 1972. Social Preference Orderings and Majority Rule. Econometrica 40, 147-57.

Downs, A., 1957. An Economic Theory of Democracy, HarperCollins, New York.

Duggan, J. 2007. Equilibrium Existence for Zero-sum Games and Spatial Models of Elections. Games and Economic Behavior, 60, 52-74.

Duggan, J., Jackson, M., 2006. Mixed Strategy Equilibrium and Deep Covering in Multidimensional Electoral Competition. mimeo, University of Rochester.

Ellsberg, D., 1961. Risk, Ambiguity and the Savage Axioms. Quarterly Journal of Economics 76, 643-669.

Gilboa, I., Schmeidler, D., 1989. Maxmin Expected Utility with Non-Unique Prior. Journal of Mathematical Economics 18, 141-153.

Ghirardato P., 2004. Defining Ambiguity and Ambiguity Attitude. In: Gilboa I. (Ed.), Uncertainty in Economic Theory: A Collection of Essays in Honor of David Schmeidler's 65th Birthday. Routledge Publishers.

Ghirardato, P., Maccheroni, F., Marinacci, M., Siniscalchi, M., 2003. A subjective Spin on Roulette Wheels, Econometrica 71, 1897-1908.

Ghirardato, P., Katz, J., 2006. Indecision Theory: Quality of Information and Voting Behavior. Journal of Public Economic Theory 8, 379-399.

Grandmont, J.-M., 1978. Intermediate Preferences and the Majority Rule. Econometrica $46,535-572$.

Hix, S., 1999. Dimensions and Alignments in European Union Politics: Cognitive Constraints and Partisan Responses. European Journal of Political Research 35, 69-106.

Hix, S., Noury, A. and Roland, G., 2006. Dimensions of Politics in the European Parliament. American Journal of Political Science 50, 494-511.

Hotelling H., 1929. Stability in Competition. Economic Journal 39, 41-57. 
Keller, R., Sarin, R., Sounderpandian, J., 2002. An Examination of Risk and Ambiguity Aversion in Decision Made by Dyads. UCLA Decisions, Operations, and Technology Management Working Paper kell001.

Klibanoff, P., Marinacci, M., Mukerji, S., 2005. A Smooth Model of Decision Making Under Uncertainty. Econometrica 73,1849-1892.

Laver, M., Benoit, K., Garry, J., 2003. Extracting Policy Positions from Political Texts Using Words as Data. American Journal of Political Science 97, 311-331.

Laver, M., Garry, J., 2000. Estimating Policy Positions from Political Texts. American Journal of Political Science 44, 619-634.

Levy, G., 2004. A Model of Political Parties. Journal of Economic Theory 115, 250-277.

Maccheroni, F., Marinacci, M., Rustichini, A., 2006. Ambiguity Aversion, Robustness, and the Variational Representation of Preferences. Econometrica 74, 1447-1498.

McKelvey, R., 1979. General Conditions for Global Intransitivities in Formal Voting Models. Econometrica 47, 1085-1112.

Mukerji, S., Tallon, J.-M., 2004. An Overview of Economic Applications of David Schmeidler's Models of Decision Making under Uncertainty. In: Gilboa I. (Ed.), Uncertainty in Economic Theory: A Collection of Essays in Honor of David Schmeidler's 65th Birthday. Routledge Publishers.

Plott, C., 1976. A Notion of Equilibrium and its Possibility Under Majority Rule. American Economic Review 57, 787-806.

Poole, K. T. and Rosenthal, H. 1997. Congress: A Political-Economic History of Roll Call Voting. Oxford University Press.

Poole, K. T. and Rosenthal, H. 1997. 2007. Ideology and Congress. New Brunswick, NJ: Transaction Publishers.

Rae, D., Taylor, M., 1971. Decision Rules and Policy Outcomes. British Journal of Political Science 1, 71-90.

Roemer, J., 1999. The Democratic Political Economy of Progressive Income Taxation. Econometrica 67, 1-19. 
Roemer, J., 2001. Political Competition: Theory and Applications, Harvard University Press, Cambridge, MA.

Simon, L., Zame, W., 1990. Discontinuous Games and Endogenous Sharing Rules. Econometrica 58, 861-872.

Schmeidler, D., 1989. Subjective probability and expected utility without additivity. Econometrica 57, 571-587. 
ftbpF 437.9375pt256.3125pt0ptFigure ftbpF5.9032in3.8147in0ptFigure

ftbpF 437.9375pt256.3125pt0ptFigure ftbpF437.9375pt256.8125pt0ptFigure ftbpF437.9375pt256.3125pt0ptFigure 